\title{
Electronic Nose and Its Applications: A Survey
}

\author{
Diclehan Karakaya Oguzhan Ulucan Mehmet Turkan \\ Department of Electrical and Electronics Engineering, Izmir University of Economics, Izmir 35330, Turkey
}

\begin{abstract}
In the last two decades, improvements in materials, sensors and machine learning technologies have led to a rapid extension of electronic nose (EN) related research topics with diverse applications. The food and beverage industry, agriculture and forestry, medicine and health-care, indoor and outdoor monitoring, military and civilian security systems are the leading fields which take great advantage from the rapidity, stability, portability and compactness of ENs. Although the EN technology provides numerous benefits, further enhancements in both hardware and software components are necessary for utilizing ENs in practice. This paper provides an extensive survey of the EN technology and its wide range of application fields, through a comprehensive analysis of algorithms proposed in the literature, while exploiting related domains with possible future suggestions for this research topic.
\end{abstract}

Keywords: Artificial intelligence, machine learning, pattern recognition, electronic nose (EN), sensors technology.

\section{Introduction}

All kinds of innovation are possible with inspiration. As image processing is inspired by the sense of sight, the electronic nose (abbreviation EN, enose, e-nose) - also known as an odor sensor, aroma sensor, mechanical nose, flavor sensor, multi-sensor array, artificial nose, odorsensing system, electronic olfactometry ${ }^{[1]}$ - technology is inspired by the sense of smell. The olfactory system easily enables living beings to be aware of their environment, of possible dangers, and to identify and classify food ${ }^{[2]}$. In technology though, automatic identification and classification of odor is a very challenging issue because the scents in chemical mixtures intercommunicate naturally ${ }^{[3]}$. This natural interaction has three types: synergism, compensation and masking. Synergism is defined as the interaction when two or more distinct substances produce a mutual scent which is stronger than those of individual components. Compensation is the case when one component counteracts another constituent. Masking is the combination of one pleasant odor with an unpleasant one. Even though there exist reported achievements of some earlier techniques ${ }^{[4-8]}$, such chemical mixtures in general conditions were not able to be analyzed and split up into its components with high accuracy until the development of the EN technology. Together with the development of EN devices, several studies were completed to assess odor intensities, to understand mixtures of odor interactions and the sensor responses to these interactions, e.g., [9-11].

Although the first research on detecting distinctive smells began in [4] during the 1920s, the idea to detect

Review

Manuscript received August 5, 2019; accepted November 15, 2019 ; published online December 28, 2019

Recommended by Associate Editor Jyh-Horng Chou

(C) The Author(s) 2019 aromas with a chemical electronic sensor array was primarily mentioned in [12] and then in [13] in the early 1980s. However, the EN concept could not be actualized at that time due to limitations in the sensors technology. In the late 1990s then, the term "electronic nose" was mentioned in [14]. According to its initial definition, an $\mathrm{EN}$ is composed of a multisensor array responsible for detect- ing more than one chemical component. Subsequently, both technological improvements in sensors and the realization of the potential that the EN holds led to a considerable extension of its applications. Recently, due to the provision of reliable solutions, rapidity, low cost and compactness, the EN concept has become popular in agriculture ${ }^{[15]}$, the food and water industry ${ }^{[16]}$, medicine $^{[17]}$, security systems ${ }^{[15]}$ and many other areas ${ }^{[18]}$.

Fig. 1 demonstrates the similarities between the biological olfactory system and the EN technology. The electronic sensor array of the EN corresponds to the olfactory nose receptors, which detects the traces of chemicals in the air. When these molecules are sensed and captured, the input signal is sent to the olfactory bulb, where the odor information is processed. After characterizing the aroma, the smell is recognized in the brain by the olfactory cortex as a last stage in the biological system. Likewise, in the EN case, a preprocessor applies feature ex-

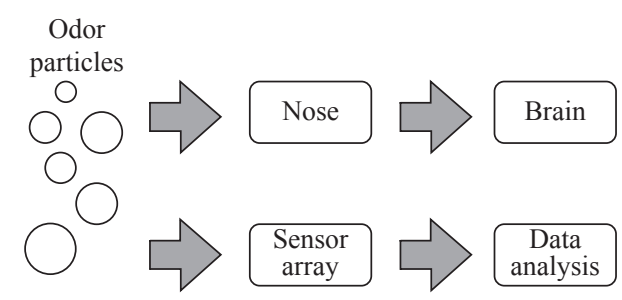

Fig. 1 Analogy between the biological olfactory system and the EN technology 
traction to the captured odor signal. Afterwards, data analysis, pattern recognition and machine learning related algorithms are generally used for identifying and classifying the input scent using the extracted digital signatures.

After drawing the parallels between the biological olfactory system and the EN technology, an EN obviously consists of both hardware and software components. While the software part can be thought as the "brain", the hardware part can be seen as the "olfactory receptors" of the EN system. The software part mainly contains a data processing unit which identifies and classifies each individual scent detected using digital signatures of the sensed chemicals. The hardware part is basically a sensor array. Since the main objective of the EN is detecting and classifying multiple aromas, the sensing array should encompass different types of individual sensors, where each sensor is responsible for detecting a different chemical. The selection of suitable sensors to a given specific task is a key point in this technology. It can be concluded here that choosing the suitable hardware and efficient software components is very important in designing and implementing a successful EN system for a particular problem. Hence, the main aim of this survey is to provide a complete overview of the EN technology while pointing out the task-dependent importance of its hardware and software components and their characteristic properties. Note that there are several existing reviews in the literature which focus on a specific sub-topic of the EN concept, e.g., the range of sensors as hardware components used in the EN systems ${ }^{[19]}$, ENs for the food industry ${ }^{[20]}$, neural networks as software components for $\mathrm{ENs}^{[21]}$. On the other hand, this paper rather provides a comprehensive review of broad EN application fields, a wide range of software related algorithms and commonly utilized sensor types and their properties as practical hardware components.

The remaining part of this survey is structured as follows. The EN device and its main components are introduced in Section 2. Then, a wide range of practical EN applications and related topics are described in Section 3. Afterwards, current challenges for this technology are discussed in Section 4, followed by perspectives and possible future research directions in Section 5. This paper is finally concluded with a brief conclusion in Section 6 .

\section{Components of an EN}

An EN consists of both hardware and software components as briefly depicted in an odor classification application in Fig. 2. Initially, the released ambient gas is absorbed by the sensor array. The detection of the input signal occurs according to the change in voltage, current, frequency, resistance parameters depending on the types of components in the sensor array. Since distinct types of sensors are usually employed in sensor arrays, the ob- tained signals should be preprocessed to understand those physical changes properly and then processed to digitalize them in order to form a dataset. Hence, the sensed signals are appropriately manipulated, e.g., amplified, filtered or converted, in order to be easily used in further stages $^{[22]}$. The processed signals are later analyzed in terms of their specific properties in the data gathering stage. Subsequently, sufficient data is acquired from these signals and the obtained data is preprocessed according to the requirements of the employed pattern recognition algorithm. Lastly, the odor is classified with the pattern recognition stage.

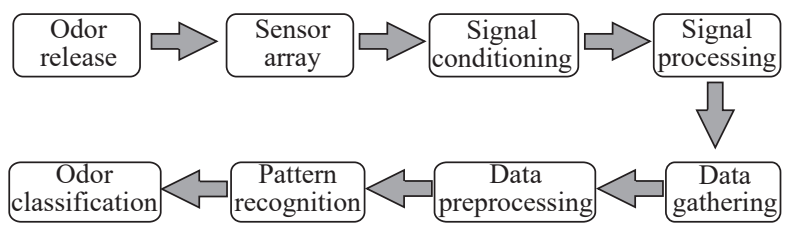

Fig. 2 A description of EN including both hardware and software components

\subsection{Sensors and chemicals}

The olfactory system can neither detect nor identify scents without the obtaining of chemicals released by the objects ${ }^{[23]}$. These chemicals can be found in simple or complex structures. Nevertheless, each individual chemical has its own unique quality and characteristics. Thus, digital signatures of chemicals, which are to be the input data for both the olfactory system and the EN instrument, are exclusive. The automatic detection process cannot be achieved without a collected library (dataset) of the digital signatures of specific aromas.

The sensor array is responsible for detecting targeted chemicals in a medium. Each targeted aroma is detected with a specific sensor, in other words each individual sensor is responsible for sensing a specific type of aroma. Chemical sensors are used for detecting chemicals in the medium. These sensors basically convert chemical information into analytical signals ${ }^{[24]}$.

Since the main objective of an EN is to sense more than one chemical, this aim can be achieved with higher accuracy only by combining several distinct sensors in the array. While keeping in mind that the sensors in the array have to be chosen carefully by taking the chemicals of interest into account ${ }^{[25]}$, having a proper sensor array for specific tasks depends on several conditions as follows ${ }^{[26]}$ : 1) small size and easy to use; 2) re-usability and inexpensiveness; 3) short response time and regeneration time; 4) high resistance for different mediums; 5) insensitivity to temperature and humidity; 6) high sensitivity and selectivity with respect to chemical compounds or substance including in the gas mixtures; 7) high stability for the selected application. 
There are numerous numbers of sensor types utilized in the EN technology. This paper summarizes below the most commonly used ones which have been applied in various practical applications, and their properties including advantages and disadvantages are given in Table 1.

\subsubsection{Metal-oxide sensors (MOS)}

MOS is the most commonly used sensor type in the EN instrument because of its suitability for wide range of gases $^{[27]}$. The practical application areas of MOS based ENs are mainly related to quality control, monitoring process, aging, geographical origin, adulteration, contamination and spoilage of food and beverages ${ }^{[28]}$. These sensors can operate at high temperatures but require high power consumption. On the other hand, oxide surface reactions are too slow at lower temperatures. The oxidative chemical reactions are constrained due to the low vapor pressure of water molecules at temperatures below $100{ }^{\circ} \mathrm{C}^{[29]}$. MOS are categorized into two main groups according to their responses to different gases ${ }^{[19]}$ : 1) $n$-type and 2) $p$-type. The operational principle of $n$-type sensors is based on the reactions between the oxygen molecules in the air and the surface of these sensors. As a result of these reactions, free electrons on the surface are trapped resulting in potential barriers between grains which inhibit the carrier mobility that produces large resistance areas. The $p$-type sensors respond to oxidizing gases, remove electrons and produce holes. Their characteristic surface reactivity and oxygen absorption significantly increase the performance of the sensor, while enhancing the recovery speed, boosting the gas selectivity and reducing the humidity dependence of signals. These sensors are fre- quently chosen in many EN applications because of their high sensitivity and selectivity[30].

\subsubsection{Conducting polymer sensors}

Although there are some drawbacks such as the inability of detecting gases like trimethylamine in fish odor applications, conducting polymers are a reliable sensor type used in many EN instruments for medical, pharmaceutical, food and beverage industries, because of their low cost, fast response to odorants and resistance to sensor poisoning[19]. Since the active layers are crucial parts of sensors, there are many different manufacturing processes of conducting polymer films including vapor deposition polymerization, thermal evaporation, electrochemical deposition, layer-by-layer (LbL) self-assembly technique, well-known Langmuir-Blodgett (LB) technique, and more ${ }^{[31]}$.

Several conducting polymers are used in sensors such as polypyrrole, polyaniline and polythiophene ${ }^{[31]}$. When a change occurs in the material due to an interaction with an analyte, the resistance in the sensor changes which leads to the detection of gases.

\subsubsection{Quartz crystal microbalance (QCM) sensors}

QCM sensors are chosen as an EN component in several applications including medicine, environment monitoring, security and food safety because of their sensitivity, convenience, rapidness, stability and portability. In these sensors, the surfaces are covered with a sensitive coating ${ }^{[19]}$. A selective barrier on the crystal surface takes in the released gas from the environment, which then increases the total mass. Subsequently, frequency decreases because of the mass change on the gold surface of the

Table 1 Sensor types and their properties

\begin{tabular}{|c|c|c|c|c|}
\hline Sensor type & Detection range & Usage area & Advantages & Disadvantages \\
\hline Metal oxide & $5-500 \mathrm{ppm}$ & $\begin{array}{l}\text { Food and beverage industry } \\
\text { Indoor and outdoor monitoring }\end{array}$ & $\begin{array}{l}\text { Suitable to range of gases } \\
\text { Operation in high temperature } \\
\text { High power consumption } \\
\text { Fast response, small size, easy to use }\end{array}$ & $\begin{array}{l}\text { High sensitivity and specificity } \\
\text { Sulphur poisoning } \\
\text { Weak precision } \\
\text { Humidity sensitive }\end{array}$ \\
\hline $\begin{array}{l}\text { Conducting } \\
\text { polymer }\end{array}$ & $0.1-100 \mathrm{ppm}$ & $\begin{array}{l}\text { Medical industry } \\
\text { Pharmaceutical industry } \\
\text { Food and beverage industry } \\
\text { Environmental monitoring }\end{array}$ & $\begin{array}{l}\text { Sensitive to range of gases } \\
\text { Fast response and low cost } \\
\text { Resistant to sensor poisoning } \\
\text { Use at room temperature }\end{array}$ & $\begin{array}{l}\text { Humidity sensitive } \\
\text { Temperature sensitive } \\
\text { Limited sensor life } \\
\text { Affected from drift }\end{array}$ \\
\hline $\begin{array}{l}\text { Quartz crystal } \\
\text { microbalance }\end{array}$ & $\begin{array}{c}1.5 \mathrm{~Hz} / \mathrm{ppm} \\
1 \mathrm{ng} \text { mass change }\end{array}$ & $\begin{array}{l}\text { Pharmaceutical industry } \\
\text { Environmental monitoring } \\
\text { Food industry } \\
\text { Security systems }\end{array}$ & $\begin{array}{l}\text { Good sensitivity } \\
\text { Low detection limits } \\
\text { Fast response }\end{array}$ & $\begin{array}{l}\text { Hard to implement } \\
\text { Poor signal-to-noise ratio } \\
\text { Humidity sensitive } \\
\text { Temperature sensitive }\end{array}$ \\
\hline Acoustic wave & $100-400 \mathrm{MHz}$ & $\begin{array}{l}\text { Environmental monitoring } \\
\text { Food and beverage industry } \\
\text { Chemical detection } \\
\text { Automotive industry }\end{array}$ & $\begin{array}{l}\text { Small size and low cost } \\
\text { Good sensitivity and response time } \\
\text { Response to nearly all gases }\end{array}$ & $\begin{array}{l}\text { Hard to implement } \\
\text { Temperature sensitive } \\
\text { Poor signal-to-noise ratio }\end{array}$ \\
\hline $\begin{array}{l}\text { Electro- } \\
\text { chemical }\end{array}$ & $\begin{array}{l}0-1000 \mathrm{ppm} \\
\text { adjustable }\end{array}$ & $\begin{array}{l}\text { Security systems } \\
\text { Industrial applications } \\
\text { Medical applications }\end{array}$ & $\begin{array}{l}\text { Power efficient and robust } \\
\text { High range operation temperature } \\
\text { Sensitive to diverse gases }\end{array}$ & $\begin{array}{l}\text { Large size } \\
\text { Limited sensitivity }\end{array}$ \\
\hline $\begin{array}{l}\text { Catalytic } \\
\text { bead }\end{array}$ & Large scale & $\begin{array}{l}\text { Environmental monitoring } \\
\text { Chemical monitoring }\end{array}$ & $\begin{array}{l}\text { Fast response } \\
\text { High specificity for combustible gases }\end{array}$ & $\begin{array}{l}\text { Operate in high temperature } \\
\text { Only for compounds with oxygen }\end{array}$ \\
\hline Optical & $\begin{array}{l}\text { Change with } \cdots \\
\text { light parameters } \\
\text { low ppb }\end{array}$ & $\begin{array}{l}\text { Biomedical applications } \\
\text { Environmental monitoring }\end{array}$ & $\begin{array}{l}\text { Low cost and light weight } \\
\text { Immune to electromagnetic } \\
\text { interference } \\
\text { Rapid and very high sensitivity }\end{array}$ & $\begin{array}{l}\text { Hard to implement } \\
\text { Low portability } \\
\text { Affected by light interference }\end{array}$ \\
\hline
\end{tabular}


QCM. Therefore, QCM sensors determine small variations on the sensor surface by measuring the frequency changes on the quartz crystal resonator ${ }^{[32]}$.

QCM sensors can operate in gas and liquid environments to determine the properties of chemicals ${ }^{[33]}$. Along with sensitive biosensors, they can detect even a nanogram of substance ${ }^{[34]}$. Importantly, QCM sensors are open for changes and improvements. The surface of these sensors can be modified so that they can detect entire cells or only one single molecular monolayer. Sensor enhancements can be actualized by an electrochemical approach, via immobilization of the silver electrode surface polyaniline film ${ }^{[35]}$. Furthermore, the biotoxicity of metallic electrodes caused by some bioactive coating films can be eliminated through employing electrodeless crystal configurations ${ }^{[36]}$. Additionally, molecularly imprinted polymers (MIPs) ${ }^{[37]}$, polished gold films ${ }^{[38]}$, biomimetic peptide-based sensing materials ${ }^{[39]}$, multi-wall carbon nanotubes $^{[40]}$, acidized-multiwalled carbon nanotubes ${ }^{[41]}$ and calixarenes $^{[42]}$ can be used in QCM sensors to coat surface layers.

As a last note, QCM based biosensors are commonly adopted to analyze odors because of their economic and easy manufacturing, and rapid analysis ability ${ }^{[43]}$.

\subsubsection{Acoustic wave sensors}

The first study about these sensors was in [44]. There are several acoustic wave sensor types, such as flexural plate wave device, fiber acoustic wave sensor, tube acoustic wave device, transverse wave device, bulk acoustic wave (BAW) and surface acoustic wave (SAW) sensors. BAW and SAW are frequently utilized in EN applications because of their small size, sensitivity, low cost and response to nearly all gases ${ }^{[45]}$. BAW sensors operate in the same way as QCM sensors, but they are less sensitive. They can measure extremely small frequency changes by means of their stability. Low cost, simplicity and robustness are other advantages of BAW sensors. However, the performance of these sensors in a liquid medium is inadequate. SAW sensors are derived from BAW devices. High sensitivity is achieved by operating at high frequencies. However, the signal-to-noise performance is poor in SAW sensors because of the high operating frequencies ${ }^{[45]}$. 2.1.5 Electrochemical (EC) gas sensors

Power efficiency, robustness, operation temperatures and ability to sense diverse types of gases are the main reasons for selecting and utilizing EC sensors in EN applications ${ }^{[46]}$. In these sensors, electrochemical oxidation or reduction of molecules occurs on a catalytic electrode surface. The amount of gas is calculated by measuring the current flow ${ }^{[7]}$. Security and industrial monitoring applications generally utilize EC sensors.

\subsubsection{Catalytic bead (CB) sensors}

$\mathrm{CB}$ is another sensor type in the EN technology. These sensors basically burn the gas of interest. As a result, a combustion enthalpy is obtained which leads to the detection of gases even in limited amounts of samples ${ }^{[47]}$.
Especially combustible gases can be identified by $\mathrm{CB}$ sensors.

\subsubsection{Optical sensors}

Optical sensors are attractive for use in several EN applications, e.g., [48], because of their compactness, immunity to electromagnetic interference and rapidity ${ }^{[19]}$. Fluorescence, optical layer thickness, colorimetric dye response, light polarization and absorbance are measured by optical sensors, and any of these optical changes are used to detect odors in the environment ${ }^{[7]}$. There are two special types of optical sensors, which detect subjects based on the differences of color, i.e., colorimetric sensors, and light, i.e., fluorescence sensors. Colorimetric sensors are composed of thin films made of chemically responsive dyes. Fluorescence sensors which are more sensitive than colorimetric sensors, identify fluorescent light emissions released by samples.

\subsubsection{Photoionization detector (PID) sensors}

By using the ultraviolet light, via high energy photons, the molecules of the targeted gas start to ionize. As a result of this ionization, formed ions create an electric current which can be detected by PID sensors at different concentration levels (parts per million (ppm) or parts per billion $(\mathrm{ppb}))^{[49]}$. Although it was clearly stated in [18] that " $\cdots$ photo-ionization detector (PID) and quadrupole fingerprint mass spectrometers (QFMS) are not considered ENs in the strictest sense because they do not provide a collective data output from a sensor array and are designed to detect and identify individual components of a gas mixture.", the PID type sensors are able to detect individual compounds and are currently used in the EN systems ${ }^{[50]}$.

\subsection{Machine learning (ML) algorithms}

"The capability of a computer to learn without being explicitly programmed for a particular task" is defined as machine learning (ML) ${ }^{[51]}$ and it was coined in 1959 ${ }^{[52]}$. ML algorithms can be grouped into four groups: 1) supervised learning; 2) semi-supervised learning; 3) unsupervised learning; 4) reinforcement learning ${ }^{[51-54]}$. In supervised learning, a dataset with known (labeled) inputs and outputs (labels) is provided to the ML algorithm. The aim is to obtain a robust mapping function (or model) between inputs and outputs. This mapping function or model will later enable the system to predict the outputs for never seen new input data. Classification, regression and forecasting are common problems in which supervised learning is generally applied. Semi-supervised learning contains both labeled and unlabeled data in the dataset. The ML algorithm learns from the labeled data, then supervised and/or unsupervised learning methods can be used to identify unlabeled samples. Semi-supervised ML algorithms are practical to use in problems with a large amount of unlabeled data to reduce computational complexity and save time. Unsupervised learning uses a giv- 
en dataset which contains only inputs. The ML algorithm investigates the dataset to learn inherent structures from this input data, trying to identify specific patterns by looking into correlations and relationships between data points. Clustering, association and dimensionality reduction problems make use of unsupervised learning. Finally, reinforcement learning is an adaptive approach in ML that learns from its past experiences and automatically determines the ideal behavior of the system while maximizing its performance for a specific task. When a new input sample is observed, the system evaluates it and produces an accurate output.

A general block diagram of ML consists in data collection, modeling, training and evaluation stages as illustrated in Fig. 3. A related dataset needs to be collected in general for every ML study. The desired successful solution cannot be obtained without a dataset which is correlated to the problem at hand. Therefore, either a precollected or a custom-built dataset is always required. After gathering a proper dataset confirming the information and specifications related to the study, a model is designed by using ML algorithms. In this modeling step, the parameter selection process is indeed crucially important for obtaining successful accuracy rates. After the training procedure, the system can be evaluated via several methods such as cross-validation.

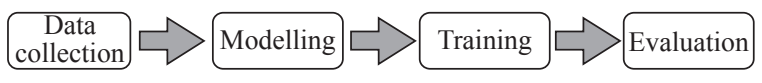

Fig. 3 General block diagram of machine learning algorithms

In the EN technology, ML is widely used due to its ability to process and comprehend large amounts of data, calibrate the gas sensor array and provide accurate classification and recognition results. Among the broad range of ML algorithms, many of them are utilized in diverse parts of the EN technology[55]. Pattern recognition algorithms are generally used for quantifying and classifying chemicals detected by an EN. Furthermore, classification algorithms are usually combined with data analysis techniques to recognize distinct aromas.

\subsubsection{Pattern recognition}

The process of identifying individual patterns through an ML algorithm is defined as pattern recognition[56]. Classification is then possible with the data acquired from these identified patterns. In other words, pattern recognition is a generic term for the ability of recognizing (ir)regularities or specific patterns in the data, whereas classification is an example of pattern recognition in which a trained model separates the data into classes. In a supervised setting, classifiers are modeled and trained using the collected training data to identify the correct class label of the object of interest. In a unsupervised learning setting, similar hidden groups are detected in the unlabeled data $^{[57]}$. Several learning based approaches are used in pattern recognition algorithms of ENs and are essential in improving EN systems ${ }^{[58]}$. Some frequently used tools include linear discriminant analysis (LDA) ${ }^{[59]}$, discriminant function analysis (DFA)[60], stepwise discriminant analysis (SDA) ${ }^{[61]}$, partial least squares regression (PLSR) ${ }^{[62,63]}$, generalized least squares regression (GLSR)[64, 65], multiple linear regression (MLR) $)^{[6]}$, principle component analysis $(\mathrm{PCA})^{[60,67]}$, support vector machines $(\mathrm{SVMs})^{[68,69]}$ and artificial neural networks (ANNs) ${ }^{[70-72]}$. The most frequently utilized data analysis algorithms for different EN sensor types are summarized in Table 2, and algorithm properties including advantages and disadvantages are given in Table 3 .

Table 2 Sensor types and frequently used data analysis methods

\begin{tabular}{cc}
\hline Sensor type & Data analysis method(s) \\
\hline Metal oxide & PCA, ANNs, discriminant analysis, \\
regression \\
Conducting polymer \\
$\begin{array}{c}\text { Quartz crystal } \\
\text { microbalance }\end{array}$ & PCA, ANNs, discriminant analysis \\
Acoustic wave & PCA, ANNs, discriminant analysis \\
Electro-chemical & PCA, ANNs, discriminant analysis \\
Catalytic bead & Pattern recognition methods \\
Optical & PCA \\
\hline
\end{tabular}

2.2.2 Principle component analysis (PCA)

PCA is a traditional and widely used unsupervised tool to reduce the dataset dimensionality linearly. It retains the variance structure of the data up to rotation. The high-dimensional data is represented in a new lowerdimensional subspace which is spanned by the principal components of largest variance in the original variables. These principal components are designated as the eigenvectors of the data covariance matrix. The highest eigenvalue in magnitude and its corresponding eigenvector retain the maximum variation, hence the most important contribution among dimensions. PCA is widely used in EN applications, e.g., [73-78].

\subsubsection{Linear discriminant analysis (LDA)}

LDA is a linear transformation technique for dimensionality reduction. It differs from PCA in the sense that LDA is a supervised method aiming at maximizing the class discrimination while taking the class labels into consideration. It works better for larger multiple-class datasets in which separability of individual classes is an important feature in reducing the data dimensionality. Some example EN applications of LDA can be found in [79-84].

\subsubsection{Support vector machines (SVMs)}

SVMs can be utilized for both regression and classification problems. As a supervised learning technique, they are widely applied in linear and nonlinear binary classification problems ${ }^{[85,86]}$. SVMs basically determine a best fitting hyperplane (i.e., decision boundary) that assist to classify data points distinctly. In order to obtain the best hyperplane, the margin between different data classes is 
Table 3 Data analysis methods and their properties

\begin{tabular}{|c|c|c|}
\hline Algorithm & Advantages & Disadvantages \\
\hline PCA & $\begin{array}{l}\text { Reduce the data dimensionality } \\
\text { Provide set of uncorrelated components } \\
\text { Measure probability estimations of high-dimensional data }\end{array}$ & $\begin{array}{l}\text { High computational time with large } \\
\text { amount of data }\end{array}$ \\
\hline Discriminant analysis & $\begin{array}{l}\text { Easy to use } \\
\text { Fast in classification applications } \\
\text { Linear decision boundary }\end{array}$ & $\begin{array}{l}\text { High computational time for training } \\
\text { Complex matrix operations } \\
\text { Gaussian assumption }\end{array}$ \\
\hline Regression & $\begin{array}{l}\text { Easy to use } \\
\text { Usage in several cases } \\
\text { Great with small amount of data }\end{array}$ & $\begin{array}{l}\text { Outliers can cause problems } \\
\text { Over-fitting occurs }\end{array}$ \\
\hline ANNs & $\begin{array}{l}\text { Can work with incomplete knowledge } \\
\text { Fault tolerance is high } \\
\text { Parallel processing } \\
\text { Once trained, predictions are fast } \\
\text { Effective with the large amount of data } \\
\text { For both regression and classification }\end{array}$ & $\begin{array}{l}\text { Hardware dependent computational time } \\
\text { Hard to find optimal network structure } \\
\text { Over-fitting may occur }\end{array}$ \\
\hline
\end{tabular}

maximized and the closest data points from both classes to the hyperplane are defined as support vectors. SVMs are commonly preferred in EN related applications ${ }^{[82,87-89]}$. 2.2.5 Artificial neural networks (ANNs)

ANNs are inspired by the working principles of biological neural networks. They are generally designed as fully-connected multilayer networks and an example structure is given in Fig. 4. The number of hidden layers changes depending on the task to be accomplished. The input layer and weights between the input and hidden layers determine the activations of hidden layers ${ }^{[90]}$. Similarly, hidden layers and weights between them decide on the activation of the output layer. It is worth mentioning here that the activation functions of individual neurons are the gates of ANNs and without them any network behaves like a linear regression model. Although there exist linear functions, nonlinear activations are generally chosen to assist the network to learn complex data structures and complex functional mappings to make solid predictions. Another reason to choose nonlinear functions is that they are more suitable to be used in back-propagation. As illustrated in Fig.5, some example activation functions include identity (linear), binary step, sigmoid (logistic), hyperbolic tangent (TanH) and rectified linear unit (ReLU). While TanH and sigmoid functions have widely been used previously, today ReLU is commonly chosen because of its pace and better performance. Moreover, ReLU can be employed where TanH and sigmoid cannot be used in networks with multiple layers due to their vanishing gradient problem. The updated version of ReLU is Leaky ReLU that prevents the dying ReLU problem for the negative input values.

Being rather than a specific algorithm, ANNs indeed constitute a "machine learning framework" in order to process data and present highly accurate results during volatile chemical substance recognition for environmental

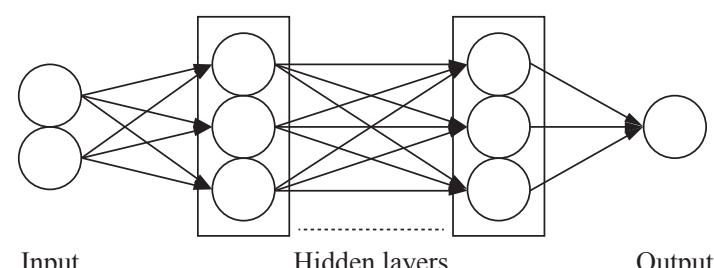

Input

Hidden layers

Output

Fig. 4 An example structure of ANNs

\begin{tabular}{cc}
$\begin{array}{c}\text { Activation } \\
\text { function }\end{array}$ & $\begin{array}{c}\text { Mathematical } \\
\text { representation }\end{array}$ \\
\hline Identity & $f(x)=x$ \\
Binary & $f(x)= \begin{cases}0, & x<0 \\
1, & x \geq 0\end{cases}$ \\
Step & $f(x)=\frac{1}{1+\mathrm{e}^{-x}}$ \\
TanH & $f(x)=\frac{2}{1+\mathrm{e}^{-2 x}}-1$ \\
ReLU & $f(x)=\left\{\begin{array}{l}0, \quad x<0 \\
1, \quad x \geq 0\end{array}\right.$
\end{tabular}

Fig. 5 Activation functions for individual neurons in ANNs

and medical purposes ${ }^{[21]}$. Therefore, ANNs and their recent extensions have become a common choice in diverse EN applications when trained with datasets containing sufficient and decent samples. Just as the data is fed to the system, trained ANNs can easily identify individual scents in a chemical mixture. However, the construction 
of a robust system requires large volumes of datasets during the training process.

In a gas sensor array, it is essential to employ a paradigm which can combine individual sensor outputs and present a comprehensive identification and classification result. Furthermore, the provision of sensor fusion enables the EN to make decisions with minimum error. ANNs contain the ability to connect the outputs from distinct sensors ${ }^{[91]}$. Hitherto, ANNs which have been employed in sensor fusion include quickprop learning algorithms, multilayer perceptron incorporated with standard back-propagation, cascade correlation and radial basis function ${ }^{[2]}$. Moreover, there have lately been many studies on EN technology incorporated with ANNs in diverse application fields, e.g., [93, 94].

\subsubsection{Deep learning}

Deep learning is yet a relatively new type of ML as a collection of techniques for mostly learning in neural networks, and is defined as the ability of a machine to automatically detect and classify desired information in a raw dataset ${ }^{[95]}$. As an advanced extension to ANNs, deep neural networks have recently been utilized in a broad range of successful academic and industrial modeling applications. Some practical examples include image and object classification and recognition ${ }^{[96-99]}$, (medical) image and video analysis ${ }^{[100-103]}$, and speech and face recognition $^{[104-108]}$. Specifically, convolutional neural networks $(\mathrm{CNNs})^{[109]}$ proposed by Hubel and Wiesel in 1962 ${ }^{[110]}$, have been frequently used among other deep learning techniques. CNNs as layered-structures generally consist of an input layer, several convolutional layers, pooling layers, nonlinear activation layers, fully-connected layers and an output layer (typically a fully-connected ANNs which is attached to softmax and classification layers). As compared to conventional feature-based pattern recognition algorithms, feature extraction and selection are automatically carried out in the CNNs technique, hence the input data does not need to be preprocessed[111]. Recent example applications of CNNs in the EN technology include liquors classification[111], and gas classification and identification $^{[112,113]}$.

Some additional deep learning based examples include convolutional recurrent neural networks (consisting in both CNNs and recurrent neural networks (RNNs)) to perform fast gas recognition[114] and (deep) Boltzmann machines ${ }^{[115,116]}$ to overcome overlapping of odors captured by an EN in order to avoid misclassification of aromas using dimension reduction and clustering ${ }^{[117]}$.

\subsection{System performance evaluation}

In a confusion matrix, results of correct predictions (true positive (TP) and true negative $(\mathrm{TN})$ ) and incorrect predictions (false positive (FP) and false negative $(\mathrm{FN})$ ) are inserted which are helpful to calculate system performance evaluation metrics ${ }^{[118]}$. The most frequently used EN system evaluation metrics are accuracy, preci- sion, sensitivity (recall, $\mathrm{TP}$ rate), specificity ( $\mathrm{TN}$ rate) and F1-score.

Accuracy is defined as the amount of correctly predicted values over all predictions, and calculated as

$$
\text { Accuracy }=\frac{\mathrm{TP}+\mathrm{TN}}{\mathrm{TP}+\mathrm{TN}+\mathrm{FN}+\mathrm{FP}} .
$$

Precision is the value that gives information about the correctly predicted positive labels among all the positive values, and calculated as

$$
\text { Precision }=\frac{\mathrm{TP}}{\mathrm{TP}+\mathrm{FP}}
$$

Sensitivity (recall, TP rate) is the proportion of correctly classified actual positives, and calculated as

$$
\text { Sensitivity }=\frac{\mathrm{TP}}{\mathrm{TP}+\mathrm{FN}}
$$

Specificity (TN rate) is the rate of correctly determined actual negatives, and calculated as

$$
\text { Specificity }=\frac{\mathrm{TN}}{\mathrm{TN}+\mathrm{FP}} .
$$

F1-score is the harmonic mean of the sensitivity and precision, and calculated as

$$
\text { F1-score }=\frac{2(\text { Precision })(\text { Sensitivity })}{\text { Sensitivity }+ \text { Precision }} .
$$

\subsection{Commercially available ENs}

At present, commercially available ENs in the market are listed in Table 4. These commercial ENs differ in terms of both sensor types and application/usage areas. As a specific example, FOODsniffer is a mobile device which gives information related to the freshness analysis of raw meat, poultry and fish. It can also connect to any smart-phone via its own application. Another example and one of the most mentioned EN in this paper, Cyranose (SenseSigents) contains 32 sensors. It can detect and analyze a wide range of gases via PCA, Knearest neighbors (KNNs) and SVMs. Therefore, Cyranose is still used in several indoor-outdoor monitoring and medical purpose applications. Another commercial EN which is mentioned noticeably in the paper is AirSense portable electronic nose (PEN). Although PEN contains 10 different sensors, the size of the device is small. It is selected for a wide variety of purposes because of its size, pace and robustness. By using various data analysis methods, this device can distinguish up to 10 different compounds which is an attractive property for research studies. Likewise, Alpha MOS provides a decidedly powerful detection and analysis product, Heracles 
Table 4 Commercially available ENs and their usage areas

\begin{tabular}{|c|c|c|}
\hline Company & Product(s) & Usage area \\
\hline Electronic sensor technology ${ }^{[119]}$ & $\begin{array}{l}\text { Computer integrated zNose } \\
\text { Battery operated zNose } \\
\text { Portable zNose } \\
\text { Benchtop zNose }\end{array}$ & $\begin{array}{l}\text { Organic, biological and chemical compounds detection and } \\
\text { analysis }\end{array}$ \\
\hline AirSense analytics ${ }^{[120]}$ & Portable electronic nose (PEN) & $\begin{array}{l}\text { Aviation, military, logistics } \\
\text { Public security, environment } \\
\text { Hazardous chemicals and explosives detection }\end{array}$ \\
\hline Electronic Nose Co. LTD. ${ }^{[121]}$ & Electronic Nose & Environment, health-care, food and beverage \\
\hline Sensigent ${ }^{[122]}$ & $\begin{array}{l}\text { Cyranose } \\
\text { eNose Aqua } \\
\text { eNose QA }\end{array}$ & Health-care, indoor and outdoor monitoring \\
\hline FOODsniffer ${ }^{[123]}$ & FOODsniffer & Food (freshness of raw meat, poultry and fish) \\
\hline EN Pty Ltd.[124] & $\begin{array}{l}\text { E-Nose Mk3.3 } \\
\text { E-Nose Mk3.3.0 } \\
\text { E-Nose Mk4 }\end{array}$ & Environment, health-care, security \\
\hline Alpha MOS[125] & Heracles Neo & Food and beverage, packaging \\
\hline RoboScientific $^{[126]}$ & $\begin{array}{l}\text { Model } 307 \\
\text { (available in limited quantities) }\end{array}$ & General purpose analyzer \\
\hline Aryballe ${ }^{[127]}$ & $\begin{array}{l}\text { NeOse Pro } \\
\text { Aryballe EN }\end{array}$ & $\begin{array}{l}\text { Automotive, personal care and cosmetics } \\
\text { Consumer appliances, food and beverage }\end{array}$ \\
\hline Odotech $^{[128]}$ & $\begin{array}{l}\text { eNose } \\
\text { SulfNose } \\
\text { MultiNose }\end{array}$ & Environment \\
\hline FIGARO Engineering Inc.[129] & Variety of gas sensors and sensor modules & Domestic and industrial monitoring \\
\hline The eNose Company ${ }^{[130]}$ & Aeonose & Health-care \\
\hline
\end{tabular}

Neo which is employed in the food and beverage industry. Its data analysis software allows it to determine various odors with high stability and sensitivity. Heracles Neo can also analyze data samples objectively even when it is used repeatedly.

\section{Current EN applications}

With the realization of the potential of the EN technology and the increasing quantity of research established in this field, it has become popular in very distinct applications. ENs have become a common device in the food and beverage industry, agriculture and forestry, medicine and health-care, indoor and outdoor monitoring, military and civilian security, packaging, cosmetics, air transportation and many more. In the remaining part of this section, a wide range of applications of EN systems will be extensively described in more detail in terms of their hardware and software components together with system performance evaluation statistics. Most of the mentioned methods will be reported with their "accuracy" statistics, when applicable. Interested readers are recommended to refer to the related publication for more information on the other evaluation metric results.

\subsection{Food and beverage}

In the last decade, EN related research topics have significantly increased especially in the food industry ${ }^{[20]}$.
Contamination of food leads to waste of resources and identifying contamination in food products is possible via ENs. Quality assessment, shelf-life and spoilage control of food, hazardous chemicals or bacteria detection and classification in food and beverages can be achieved through some special sensor arrays. The activity of microorganisms, enzymes and fat oxidation in the food production and storage stages result in deterioration. This decline in quality may lead to the formation of harmful bacterias or nutrient loss, which can later cause important food safety issues. Fortunately, the spoiled food releases chemical gases which can be sensed by an EN.

\subsubsection{Quality assessment and adulteration of meat}

Thanks to high amounts of protein, vitamins and minerals they contain, meat and meat products are to be considered indispensable for the diet of the most people in the population. In research by the Food and Agriculture Organization of the United Nations, the global meat production rose by $20 \%$ (between 2012 and 2014) and this number will increase again by $17 \%$ in the further periods ${ }^{[20]}$. This continuous growth proves that, in the near future, meat quality assessment from production to consumption and automatic classification of meat products needs to be accomplished as fast as possible with low cost and high accuracy. However, traditional techniques mainly analyze the meat samples in special laboratories and identify the spoilage by examining the total amount of bacteria inside the meat, which is very 
time consuming.

The EN technology combined with ANNs were started at first to be used to address and overcome the above mentioned problem. In 1996, Yano et al.[131] introduced a biosensor system which monitors the meat spoilage and the aging process. Their system requires direct contact with the meat surface, an $\mathrm{AgAgCl}$ and a platinum electrode. The results demonstrated an estimation of meat quality through aging, which was then improved with several modifications.

Later in early 2000s, the EN technology proved its high potential for both food and feed industries with the study of Santos et al. ${ }^{[73]}$ An EN was proposed using tinoxide gas sensors by means of PCA and ANNs to differentiate between Iberian ham samples obtained from pigs with various feeding regimes and ripening times. Their system successfully distinguished the distinct types of hams which have different adulteration. In addition to [73], an EN with semiconductor thin film sensors was later used in [132] to discriminate four types of ham. The samples were analyzed with PCA and probabilistic ANNs concluding the beneficial aspects of the EN technology in discriminating food samples.

In [133], the relationship between the quality of the pork meat pizza topping products and storage conditions were analyzed. Moreover, the sensor quality versus storage conditions were also examined. The researchers first categorized independent data as known (i.e., production samples) and unknown (i.e., purchased samples), and then kept them at $5^{\circ} \mathrm{C}$. PLSR was used for modeling their system which predicts the storage time with respect to the sensor quality changes during the storage period. The experimental results illustrate that the storage time of both known and unknown samples were predictable as the sensor quality changes during storage. As a result, this study gives strong proof that ENs will become in future implemented devices in quality control of meat products.

A quality assessment of red meat was accomplished with metal-oxide semiconductor sensor arrays in [134]. Samples were taken from beef and sheep meat stored at $4{ }^{\circ} \mathrm{C}$ for up to 15 days. While PCA and SVMs were selected as software components, a bacteriological method was utilized for training the EN. According to this research, the observed correlation between the bacteriological data and EN signals were satisfying. Hence, the proposed EN system proved its potential to become a simple and time saving technique for the quality assessment of red meat. Additionally in [80], the meat freshness was controlled with an EN based on a metal-oxide multisensor array using the LDA algorithm. The proposed system was able to identify whether the meat was produced by the same supplier, or not, by only examining one or two samples. Moreover, the early stage of spoilage in the stored meat, at $4{ }^{\circ} \mathrm{C}$ and $25^{\circ} \mathrm{C}$, could be detected accurately by LDA. These studies demonstrate that MOS ENs are ready to be used in the food industry.

In [81], an EN was developed using a PEN 2 with ten metal-oxide sensors for the assessment of pork freshness. LDA and back-propagation ANNs were utilized for storage time prediction and MLR was used to estimate the sensory scores. While the suggested system had the prediction accuracy rate of $97.14 \%$, the correlation between EN signals and sensory scores was high. In a related study in [135], hairtail fish and pork samples stored at $5^{\circ} \mathrm{C}, 10^{\circ} \mathrm{C}$ and $15^{\circ} \mathrm{C}$ temperatures were taken for the classification of their freshness. A metal-oxide sensor array was used to sense chemicals, and pattern recognition based on PCA and compensation methods were adopted to analyze the gathered data. After simultaneously measuring the total volatile basic nitrogen and aerobic bacterial counts of samples, the proposed EN system could rapidly analyze the stage of spoilage of hairtail fish and pork with high classification accuracy rates $(87.5 \%$ for fish, $91.7 \%$ for pork).

In [136], a portable EN based on electrochemical sensors was proposed to analyze food. A successful system adopting SVMs was developed to classify poultry meat by taking into account their shelf-life with an accuracy of $100 \%$. A fast detection and identification model for poultry meat species and meat adulteration was developed using a PEN 3.5 portable EN in [137]. During the heating process at $25^{\circ} \mathrm{C}, 80^{\circ} \mathrm{C}, 100^{\circ} \mathrm{C}, 120^{\circ} \mathrm{C}$ and $150^{\circ} \mathrm{C}$ temperatures, volatile chemical samples released from chicken, duck and goose meats were collected and then analyzed by adopting LDA and DFA. As a result, the EN could recognize various heating temperatures and differentiate meat types with more than $95 \%$ accuracy. Another system to distinguish different meat species and to identify the spoilage level was developed in [138] using a sensor array which contains six metal-oxide sensors. This EN system was supported with a device based on an electronic tongue (e-tongue $)^{1}$. The meat samples were collected from beef, goat and sheep, and stored at $4^{\circ} \mathrm{C}$. Data analysis and classification tasks were performed by PCA and SVMs concluding that these three kinds of red meat can be classified successfully and also the duration spent in the storage can be identified.

In [139], a new low cost and portable EN with metaloxide sensors was designed aiming at evaluating the chicken meat freshness and microorganism population at $4^{\circ} \mathrm{C}$ and $30^{\circ} \mathrm{C}$ for up to 5 days. While PCA was employed in classification of chicken meat freshness for different storage temperatures and days, back-propagation ANNs were used to built a model for microorganism population assessment which demonstrates very accurate res-

${ }^{1}$ The International Union of Pure and Applied Chemistry Analytical Chemistry Division (IUPAC) defines electronic tongue as "The electronic tongue is a multi-sensor system, which consists of several low-selective sensors and uses advanced mathematical procedures for signal processing based on the pattern recognition $\cdots$ and/or multivariate analysis." [140] 
ults. With additional studies ${ }^{[141-144]}$, EN systems offer benefits such as pace, portability, low cost and nondestructive measurements with high relative accuracy, hence these systems can be used as an alternative for the analysis of bacterial population on meat and meat products.

A recent EN system based on three gas sensors and one color (red, green, blue) sensor array was proposed in [145] to differentiate three freshness levels of meat as fresh, half-rotten and rotten. The developed system reached accuracy up to $80 \%$ for these three freshness levels using ANNs. Authors claimed that there exists pattern resemblance between half-rotten and rotten meats, hence an error of $20 \%$ occurs. The system achieves $100 \%$ precision in the case of only distinguishing between fresh and rotten meat.

\subsubsection{Cocoa beans and chocolate}

In order to determine the roasting level in cocoa beans, ANNs are usually trained by a gas chromatography/mass spectrometry (GC/MS) system. However, this is a time-consuming, very complicated and expensive process. A new EN model aiming at determining the degree of roasting in cocoa beans was proposed in [146] by means of volatiles of cocoa beans roasted for different durations. ANNs were used to estimate the level of roasting using signals acquired from seven sensors. The developed system reached a $94.4 \%$ prediction accuracy in comparison to that of GC/MS which is $95.8 \%$.

In the chocolate industry, flavor analysis keeps an important place for the production stage ${ }^{[147]}$. The study in [147] was performed to classify regular and artificially offflavored chocolate aromas through an EN. Several samples from both classes were collected and examined via two different ENs composed of metallo porphyrins and gold nanoparticles peptide sensors. A classification accuracy of more than $90 \%$ was achieved by the gold nanoparticles peptide sensors. In a different study ${ }^{[148]}$, a self-constructed EN with seven metal-oxide sensors was adopted to differentiate 26 types of distinct chocolate samples, extra ingredients, sweetener and expiration date status. PCA and ANNs were employed as classifiers and an average identification rate of $81.3 \%$ was obtained.

\subsubsection{Alcohol and alcoholic beverages}

An EN system was utilized in [149] to identify distilled liquors with various types of pattern recognition algorithms. Twelve test samples of distilled spirits were identified using PCA and different types of ANNs (i.e., back-propagation ANNs, linear ANNs, radial basis function ANNs and self-organizing map ANNs) using eight $\mathrm{SnO}_{2}$ gas sensors in the sensor array. While reporting that the back-projection ANNs reached 100\% recognition accuracy for this test set, its slow convergence issue was solved by applying a chaotic back-projection ANNs instead. In a similar study in [111], an EN with ten metaloxide sensors was proposed for classifying seven types of Chinese liquors. The sensor responses were converted into gray-scale images and processed with a CNNs architec- ture containing two convolutional layers, two pooling layers, a fully connected layer and an output layer. The highest test classification accuracy reached was $95.7 \%$ by CNNs, when compared to SVMs (92.9\%), ANNs (91.9\%) and LDA (85.8\%).

A portable EN with twelve metal-oxide sensors was developed in [82] to classify rice wines with different marked ages. PCA, LDA and locally linear embedding (LLE) ${ }^{[150]}$ were chosen to be used in the classification step of wine samples, whereas PLSR and SVMs for the predictions of the marked ages. According to this study, LDA provides best results for classification and SVMs work much better than PLSR in the prediction stage. A portable EN based on an mbed micro-controller was proposed in [151] to identify synthetic wine samples. A sensor array was built by using four different sensors and two algorithms were adopted in the study. While ANNs presented a classification rate of over $99 \%$, SVMs reached an accuracy of over $93.5 \%$. The authors pointed out that the designed EN is very economic to built.

Meeting aroma expectations of customers is an important task for also the beer industry and exploiting the best standard for the beer aroma can be achieved by an EN. A study in [152] discriminated different types of beers with various alcohol contents through a self-developed EN based on PCA and ANNs. The beer samples were classified with $84.72 \%$ accuracy. A similar study in [153] proposed an EN system for classifying ten kinds of Thai beers. Coupled with ANNs, the system had a success rate of $87.5 \%$ in discriminating all brands. In [154], alcoholic and non-alcoholic beers were discriminated using a metal-oxide sensor system by means of ANNs with $100 \%$ accuracy. Further research on a proposed EN system for water evaluation in beer production sector can be found in [155].

\subsubsection{Tea and tea quality}

A comprehensive study was performed in [156] to analyze tea samples with an EN with a metal-oxide sensor array. The data was collected from five tea samples under different manufacturing processes, in different qualities and in the consideration of humidity and temperature. The gathered samples were processed and analyzed using PCA, fuzzy $\mathrm{C}$ means $(\mathrm{FCM})^{[157]}$ and different types of ANNs, e.g., probabilistic ANNs, radial basis function ANNs and self-organizing map ANNs. The proposed system was noticeably successful in distinguishing between these five flavors of tea samples, leading to high classification accuracy rates of $100 \%$ for radial basis function ANNs and of $94 \%$ for probabilistic ANNs. Moreover, authors emphasize that the training time of these two ANNs methods was faster when compared to the other ANNs.

Research in [158] was carried out to identify five different quality degrees of green tea samples using a PEN 2 with ten metal-oxide sensors. While PCA was used to optimize the system and reduce the dimensionality, LDA 
and back-propagation ANNs were used to analyze the processed data. Tea grading results indicate that both LDA and ANNs algorithms were more successful for tea beverages than tea leaf and tea remains. Another tea quality assessment was performed in [87] using an EN and three types of multi-class SVMs. The designed EN includes an array of four sensors, and the aromas were collected from dried black tea samples to avoid humidity. The accuracy of tested SVMs models had more than $97 \%$ to estimate various qualities of black tea. A recent study was proposed in [159] to estimate black tea flavors using a "low-cost hand-held" EN containing four metal-oxide gas sensors. The data samples were processed and investigated through PCA, self-organizing map $\mathrm{ANNs}$ and back-propagation ANNs. The designed system was successful with the accuracy of $90 \%$ over a set of 80 different tea samples collected from various places.

\subsubsection{Coffee and coffee quality}

A study was carried out in [160] for the quality assessment of seven different instant coffee types from Brazil and England. The coffee samples were collected via PEN 2 EN equipped with ten metal-oxide sensors. The proposed system was able to distinguish the evaluated coffee types with an accuracy of $100 \%$ using ANNs. Additionally, a recent study in [161] successfully classified six different instant coffee types produced by the same industry. A PEN 2 with seven metal-oxide sensors was used to gather 53 aromatic pattern profiles of coffee samples. LDA and common dimension analysis were applied to these data for classification. The proposed system was able to noticeably discriminate different instant coffee products.

Another study in [65] described a PEN 3 based EN system for online monitoring of industrial coffee roasting, for assessing the roasting degree of coffee beans originated from Brazil, Costa Rica, India and Vietnam. While the correlation between EN signals and roasting degrees was obtained with both PLSR and GLSR, it turned out to be the proposed system with GLSR that could successfully differentiate all roasted coffee beans by checking their volatile organic compound profiles. A related work based on PCA and ANNs was performed in [74] to predict coffee roasting degrees using an EN containing a metal-oxide sensor array. The developed method was able to produce high success rates for both estimating the roasting time and coffee quality parameters.

Some research was performed in [162] to determine the origins of different coffee samples based on their flavor. The Heracles II EN system was utilized and the collected samples were analyzed through PCA and DFA. Experimental validations indicate that the DFA was a successful tool for predicting the geographical origins of coffee samples.

\subsubsection{Oil and vinegar}

In contrast to gas chromatography and chemical analysis, ENs can rapidly assess edible oil quality and identi- fy the origin of oil types ${ }^{[163]}$. In addition, since deep fatfrying and storing conditions alter the quality and aroma stability of products ${ }^{[164,165]}$, it has become another field in which ENs were adopted. In [166], it was aimed to determine the quality of vegetable oils which engage with thermal treatment. A system based on ultra-fast gas chromatography along with chemometrics and flame ionization detector was designed. Although very promising results were obtained, the researchers desire to built an $\mathrm{EN}$ in order to construct a generic system to assess the quality of vegetable oils.

In [136], rapeseed oil was successfully classified using SVMs with a success rate of $100 \%$ and adulterations of extra virgin olive oil with rapeseed oil were of $82 \%$ through considering the degree of thermal degradation. Yet a QCM sensor array based EN system developed in [75] was able to classify the quality of olive oil samples from aromatic profiles using PCA. Experimental results of this easy to use and low cost approach were satisfactory for distinguishing virgin olive oils from lampante ones. A related method was presented in [167] to detect adulteration of olive oils using Cyranose 320 . The collected samples of olive oil from different regions of Balikesir in Turkey were analyzed through various algorithms such as PCA, LDA, ANNs, SVMs, KNNs. The best success rate was reported with a Naive Bayes classifier having a $70.83 \%$ accuracy. A recent study was performed in [168] to assess the quality of olive oil samples. An EN with MQ-series $\mathrm{SnO}_{2}$ gas sensor array was designed and used to collect samples. The proposed system was able to distinguish between virgin oils from pomace oils by around 90\% prediction accuracy.

In addition to olive oil, vinegar is also widely used in homes and industry; hence ENs can be employed to identify and classify different types and qualities of vinegar. For example in [169], an EN consisting of 14 tin-oxide sensors was used to identify six types of Chinese vinegar using a new kernel based DFA method. The collected vinegar samples were successfully classified by up to $100 \%$ success rate.

\subsubsection{Dairy products}

Milk requires a strict quality control due to its complicated structure and traditional methods for milk quality control are expensive, complex and sometimes subjective $^{[170]}$. In [170], a portable EN with MQ-135 (responsible to detect carbon dioxide and ammonium) and MQ-3 (responsible to detect ethanol, hexane, methane and carbon monoxide) sensor array was designed to identify mastitis milk and to estimate the milk parameters such as acidity, lactose, protein, ashes and casein content. In a recent study ${ }^{[171]}$, milk spoilage identification and diseases detection problems were addressed through an EN system with a metal-oxide sensor array. To sum up, experimental results reported in both above studies show that EN systems are beneficial in milk quality control and provide a new, rapid method for the dairy industry. 


\subsubsection{Water quality control and monitoring}

Water concerned EN applications are diverse. An example study was performed in [172] to develop an EN system for the identification of microbial and chemical contamination of potable water. The system designed was based on two different conductive polymer sensor arrays with PCA, DFA and cluster analysis, and resulted in a model effectively distinguishing between the different bacterial and fungal species. Yet another example work in [173] was put into practice in Bangkok to classify the odors of wastewater and fresh water. Wastewater samples were collected from different domestic canals with an EN with three metal-oxide sensors. The system was able to successfully detect relative amounts of organic solvent vapors, ammonia, liquid petroleum gas, hydrogen and carbon monoxide in the collected samples. In a recent study ${ }^{[174]}$, to identify scents from wastewater plants, researchers suggested an EN system based on PCA to distinguish odors emitted from wastewater treatment plant, and a PLSR model to predict the odor concentration. A successful EN application in [175] aimed at detecting oil pollution in seawater samples via six paper-conducting polymer gas sensors using ensemble machine learning classifiers. Additionally, the study in [176] proposed to monitor the early activity of Streptomyces in water. An EN system was developed with 14 conducting polymer sensor array using PCA and DFA. The developed model was able to not only detect the Streptomyces but also discriminate between species.

\subsection{Agriculture and forestry}

The EN technology is commonly used in agriculture, in which related applications are used for agronomy, horticulture, botany, biochemical procedures, pesticide detection, infestation detection, plant physiology, plant pathology, environment monitoring, and many more ${ }^{[177]}$. More specifically, fruits, vegetables, flowers, plants, tobacco and all related products release specific volatile organic compounds which can be sensed by an EN.

An investigation was performed in [178] to monitor the quality of fruits and to classify their maturity. An EN system was employed with a metal-oxide sensor array using PCA, KNNs and SVMs algorithms. The samples were collected from bananas with four different maturity states leading to an accuracy of $90 \%$ or higher for the classification of the maturity of fruits. A related study was performed in [179] to classify four different ripening stages of bananas by using an EN together with a camera. The camera system was used to capture the peel color of the fruit as an extra feature, while the EN sensor array was established from seven metal-oxide sensors. PCA, LDA, KNNs and SVMs were applied to analyze data samples and the proposed EN/camera system could distinguish the maturity stages of bananas with an accuracy rate of $100 \%$. A very recent study was proposed in [83] to pre- dict the ripeness level of post-harvest kiwifruit using PEN 3 EN. The collected aroma samples were analyzed with LDA to discriminate kiwifruits at different ripening times. Moreover, the aroma volatiles were examined through several algorithms such as random forest (RF) classifier ${ }^{[180]}$, PLSR and SVMs. The prediction model based on the RF classifier showed better performance than PLSR and SVMs, in order to estimate the overall ripeness, soluble solids content and firmness of post-harvest kiwifruits. Another fruit-based successful EN (PEN 3) related research was accomplished in [181] to distinguish the damage severity of Fuji apples. After dropping each apple from a different height onto a cement floor, the collected apple volatile compound samples were analyzed with PCA, LDA, SDA and different types of ANNs. According to reported results, LDA was more efficient than PCA for distinguishing damage severity of apples and multilayer perceptron ANNs resulted in $100 \%$ with the SDA dataset.

In [182], 584 samples of mixed vegetable soup were artificially contaminated by Enterobacter hormaechei and Escherichia coli. An EN (EOS-507C[183]) composed of four metal-oxide sensors was adopted along with the LDA algorithm. The classification accuracy of contaminated samples was $98 \%$. In another study ${ }^{[88]}$, a bionic EN with five metal-oxide sensors was employed aiming at detecting the potato tuber soft rot infection in its early stages. Soft rot is a common bacterial disease in potatoes and may cause important economic loss during storage. To address this problem, the collected data analyzed according to the maximum, average and minimum gradient features through radial basis function $\mathrm{ANNs}$ and SVMs. SVMs reached a classification accuracy of $89.7 \%$ which was superior to the ANNs algorithm. Yet another study in [184], a portable EN based on PCA was developed to identify tomato plants infested by aphids and white-flies by means of their volatile profiles. The early stage of the infestation was detected with an accuracy rate of $86 \%$.

Alkaloid degrees in tobacco products must be retained at certain levels according to regulations ${ }^{[185]}$. In [185], an EN utilized with 18 metal-oxide sensors using PCA and PLSR analysis to control nicotine alkaloid levels in tobacco. As a result of this study, it was stated that the EN device was successful in detecting the degree of nicotine alkaloids, but further study is needed to improve the robustness of the system. Another tobacco related EN proposed in [186] to distinguish cigarette brands and improve the identification rate. The smell-prints of four different cigarette leaves were collected via Cyranose 320 with 32 sensors, and these samples were analyzed through ANNs. Experimental results indicate that ANNs could greatly improve the accuracy of classification while comparing with the Cyranose 320 software results.

Forestry has also become an important application area of ENs ${ }^{[177]}$. Preventing waste in forests, processing wood and paper, monitoring and protecting the forest 
health, classifying plants and animals according to their biochemical composition are some example tasks in which ENs can be employed. An extensive review of a wide range of EN applications in agriculture and forestry can be found in [177].

\subsection{Medicine and health-care}

In traditional methods of diagnosing diverse illnesses, human-driven mistakes may occur and these approaches are generally slow, and more importantly expensive. The EN technology offers an alternative, fast and cost efficient solution to diagnose certain diseases. Moreover, health-care and well-being applications already contain a broad usage of ENs. The EN technology has confirmed its benefit in health-care with some example studies such as [187-189]. Various volatile organic compound patterns in the breath of a patient can be sensed, hence ENs are able to recognize and classify various illnesses releasing casespecific chemicals. Moreover, ENs have a promising potential in separating healthy and sick subjects with diseases of different severity[190].

Some example applications of EN in medicine are as follows. An EN system was designed in [191] to distinguish subjects with renal dysfunction from the healthy ones by means of the odor changes of human body. The proposed system contains three thick-film metal-oxide based gas sensors. The odor samples were collected from 11 healthy controls, 42 patients with end-stage renal failure (DP) and 20 with chronic renal failure (CRF). The collected samples were analyzed via PCA and quadratic discriminant analysis (QDA) leading to promising results such that the patients with CRF were distinguished from the DP with a success rate of $95.2 \%$. In [192], the agents of the Chagas disease (ECh), Trypanosoma cruzi, were investigated according to triatomines of different sexes and development stage using the Laser Vaporization EN technique. The data samples collected from the EN were analyzed with PCA and LDA. Consequently, ENs were proved to be sufficient for categorizing these parasites into their sexes and nympha stages, by checking their volatile emissions. In another study proposed in [193], the anesthetic dose level prediction was achieved through an EN with QCM sensors and ANNs. ANNs were trained with the Levenberg-Marquardt algorithm ${ }^{[194]}$ to illustrate the relationship between eight anesthetic dose levels and the frequency changes on QCM sensors. The developed system demonstrated acceptable anesthetic dose level predictions with an accuracy rate of $95 \%$.

A common disease, namely Bile acid diarrhoea (BAD) was detected in [195] by using a system based on Alpha MOS Fox-4000 EN and an Owlstone Lonestar Field Asymmetric Ion Mobility Spectrometer. LDA was adopted to analyze the collected data from 110 patients; 23 with BAD, 45 controls and 42 with ulcerative colitis (UC). According to experimental results, a classification success of $85 \%$ was reached for all groups.

The EN technology was further employed in the diagnosis of urinary tract infections (UTI). In [196], an EN system combined with ANNs was able to distinguish the patients with the disease. The data samples were obtained from 25 and 45 patients using an array of 14 sensors (RoboScientific model BH114-Bloodhound) to complete two different experiments. The results of both experiments demonstrated that ENs can be used in early detection of UTI. A related EN study in [197] also supports these promising results of the UTI disease identification.

The breath sampling and data collection through ENs is diverse in medical research studies ${ }^{[198]}$. Performing a reliable and accurate breath sampling has indeed major importance for obtaining correct analysis results ${ }^{[199,200]}$. Due to limitations in the sensors technology, some analytes might not be recognized by the sensor ${ }^{[201]}$. Usually sensors in the market are able to detect compounds at 1 ppb concentration levels, however analytes can be present at very low concentration levels ${ }^{[202]}$ which will inevitably lead to an analyte-ignorance by the sensor during the breath sampling process. Biosensors on the other hand are more sensitive and can be adopted in the medical field, but these sensor types are rarely employed in medical applications due to their high cost and difficult manufacturing process $^{[201]}$.

\subsubsection{Asthma}

In [203], the aim was to classify patients with asthma with different disease severity from healthy controls, by analyzing their smell-prints of exhaled air using an EN (Cyranose 320). The samples were collected from different ages and disease severity groups. The gathered data were analyzed using LDA on PCA. As a result, the EN could successfully differentiate exhaled breath samples taken from patients with asthma from controls. However, it was less accurate in determining severity of the disease. A related study was performed in [204] to analyze if EN systems were able to classify allergic rhinitis with and without concomitant extrinsic asthma, and healthy controls. The samples of exhaled breaths of patients were gathered with a Cyranose 320 which include patients with extrinsic asthma and allergic rhinitis, patients with allergic rhinitis without asthma and controls. The PCA and canonical discriminant analysis (CDA) based model then tested with 21 newly recruited samples. The outcomes of this study was promising with a peak cross-validation accuracy of $85.7 \%$.

A Cyranose 320 based EN system was proposed in [205] to distinguish between patients with asthma and chronic obstructive pulmonary disease (COPD) which can have overlapping clinical symptoms with asthma. The exhaled breath samples were taken from subjects including patients of COPD, patients of asthma, non-smoking and smoking controls. The system based on PCA and CDA was able to successfully discriminate asthma patients 
from COPD patients, non-smoking controls and smoking controls with accuracy rates of $96 \%, 95 \%$ and $92.5 \%$, respectively.

\subsubsection{Cancer}

A recent study was performed in [206] to detect lung cancer from the breath samples via an EN with metal-oxide gas sensors. The collected breath samples from lung cancer and healthy controls were analyzed with PCA and LDA to reduce the raw data to two-dimensions in order to explore the data distribution. The system was developed with KNNs and SVMs, resulting in the best accuracy of $84.4 \%$ by means of the PCA-KNNs method. A similar work was accomplished in [207] for detecting lung cancer with an Aeonose EN through ANNs. The breath samples were taken from lung cancer patients and healthy controls exhaled through five EN devices for five minutes. As a result of this study, an overall diagnostic accuracy of $86 \%$ was achieved for the blinded validation set.

Yet another study was proposed in [208] to diagnose head and neck cancer by means of an EN with twelve metal-oxide sensors. The exhaled breath samples were collected from patients with head and neck squamous cell carcinoma and analyzed through pattern analysis of their volatile organic compounds. The control group was selected from patients visiting the outpatient clinic for other conditions. The designed system was successful while indicating its usefulness in diagnosing the head and neck cancer.

\subsubsection{Tuberculosis}

In [209], an EN was designed with an array of conducting polymer sensors using PCA and ANNs. PCA was able to differentiate between tuberculosis infection alone, the controls, Mycobacterium avium, Pseudomonas aeruginosa and a mixed infection. Moreover, through ANNs and cross-validation, tuberculosis cultures were discriminated from others with an accuracy of $100 \%$. A similar EN was developed with 14 conducting polymers in [210] to diagnose tuberculosis in order to avoid the Ziehl-Neelsen staining procedure which is time-consuming and lacks sensitivity. 330 culture-proven samples of virus-tested tuberculosis and nontuberculosis were analyzed with PCA, DFA and ANNs. The system was able to identify the tuberculosis with an accuracy rate of $89 \%$ for culturepositive patients. An additional relatively recent research was performed in [211] to detect tuberculosis via an EN (DiagNose), i.e., an EN with metal-oxide sensors, being four different sensor types in triplicate. The exhaled air samples were collected from subjects including participants for the pilot and validation study. The ANNs based EN identified samples with tuberculosis from healthy controls with a sensitivity of $93.5 \%$ and a specificity of $85.3 \%$ in the validation step. These mentioned studies have very important conclusions for the development of fast and objective EN systems to diagnose tuberculosis via patient samples.

\subsubsection{Sinusitis}

The objective of the study in [212] was to investigate the nasal breath samples of the patients with chronic rhinosinusitis and healthy controls using the EN technology with a QCM sensors array. The developed simple technique with PCA correctly classified all individual data for chronic rhinosinusitis and misclassified two samples from the controls with a classification success rate of $80 \%$, whereas $A N N$ s had a success rate of $60 \%$. In [213], colorimetric sensor arrays were suggested to be used for distinguishing between exhaled breath from patients with and without chronic bacterial sinusitis. 11 sinusitis patients and 9 controls were selected and the collected data from the array (color changes for each time point) were classified with binary logistic regression using PCA. As a result, the accuracy of suggested method peaked at $90 \%$ which is very promising.

\subsubsection{Cystic fibrosis}

With some example studies in [214, 215], the exhaled breath samples were collected from both cystic fibrosis patients and healthy controls via an EN. Volatile organic compounds have been processed in this domain and it was concluded that breath analysis is feasible and holds potential for the diagnosis of cystic fibrosis lung disease.

\subsubsection{Smokers and non-smokers}

ENs are useful not only in disease diagnostics but also in distinguishing smokers from non-smokers by examining their breath smell-prints. A study was performed in [216] to differentiate the breath volatile organic compounds of smokers from those of non-smokers with Cyranose 320 based on PCA. The smokers were differentiated from non-smokers with a cross-validation value of $95 \%$. Another related study in [217] aimed to compare the efficiency of ENs with $\mathrm{SnO}_{2}$ and $\mathrm{WO}_{3}$ sensor arrays. Experimental results reported indicate that $\mathrm{WO}_{3}$ based EN is better than the one with $\mathrm{SnO}_{2}$ in discriminating among smokers, non-smokers and normal air samples using PCA and SVMs. In [218], an EN system was developed to detect odor changes of human body due to cannabis consumption. The samples were collected from 40 volunteers consisting of 20 cannabis-smokers and 20 tobacco-smokers. The obtained samples were analyzed through PCA with a classification success rate of $70 \%$ and SVMs with $92.5 \%$.

\subsubsection{Human space-missions}

The EN technology can be used in human deep-space missions in order to support the health of astronauts ${ }^{[219]}$. Considering small-size compact EN devices with medical diagnostic capability, it might be implemented into the spacesuits to analyze blood, breath, saliva and measure any biomarker.

\subsubsection{Drug-related fields}

An important field related to the health-care and medicine is the pharmacy. It is an obvious fact that human beings overcome various diseases with medications. Therefore, the drug production process needs to be done 
minutely. To eliminate human errors and gain pace in the production stages of these medications, ENs are used in the pharmaceutical industry in quality control, formulation studies, illegal drug detection, etc.[220] For instance, the study in [221] aimed to detect illegal drugs using a portable EN and ML algorithms, PCA and SVMs. The designed model was successful at distinguishing five different drug samples (cannabis buds, cannabis plants, hashish, snuff tobacco, tobacco leaves) and detecting illegal drugs. Moreover, the samples of Radix Angelica Sinensis collected from different locations in China were analyzed through PCA and DFA in [222] using the Fox-4000 EN. The proposed model demonstrated great results in identifying samples from different growing places.

\subsection{Indoor and outdoor monitoring}

Air pollution is one of the crucial problems in modern, developed and developing countries. Hazardous chemicals can cause serious diseases and decrease the quality of life ${ }^{[55]}$. Moreover, decreasing the unpleasant odor amount released from sewers and wastewater treatment plants is an important issue ${ }^{[223]}$. Eliminating these problems is impossible without detecting toxic gases which cause the pollution. There are various studies concerned to address these problems through ENs. For instance in [224], an EN application which was based on SAW sensors, PCA and ANNs were successfully used to detect the binary mixture of dimethyl methylphosphate (simulant of nerve agent) and methanol. An EN system was proposed in [225] to monitor and predict indoor air quality via metaloxide gas, and temperature and humidity sensors. Three ANNs models were trained with the LevenbergMarquardt algorithm and the system was able to successfully detect and predict the concentration levels of the methane and carbon dioxide gases even when there exists an interfering in-place hydrogen gas. In another related study ${ }^{[26]}$, a successful EN with three gas, temperature and humidity sensors array using back-propagation ANNs was developed to monitor indoor air quality in living environments. Statistical analysis results were higher than those of competing methods including SVMs, radial basis function ANNs and self-organizing maps. Recent research completed in [227] compares the performance of three different sensors based ENs for real-time outdoor air monitoring. The ENs were based on semi-conducting gas sensors only, amperometric gas sensors only and both type of gas sensors. SVMs were used for calibration and proved to be useful in the semiconductor sensor responses of $\mathrm{CO}, \mathrm{NO}_{2}$ and $\mathrm{O}_{3}$. Note here that there are other examples of published studies related to detection and identification of diverse odors, toxic vapors and gases (such as ammonia, acetone and ethanol) based on $\mathrm{ANNs}^{[228,229]}$.

In a further study, a 38-layer Deep CNNs (GasNet) was adopted in gas classification ${ }^{[12]}$. Compared with mul- tilayer perceptron ANNs and SVMs, CNNs provided better results however the processing time was longer. An accuracy of $95.2 \%$ was achieved with CNNs in $154 \mathrm{~s}$, while SVMs reached $79.9 \%$ in $2 \mathrm{~s}$ and ANNs reached $82.3 \%$ in 17 s. Similarly, LeNet-5 ${ }^{[230]}$ CNNs were adopted in [113] to classify gases through an EN with twelve metal-oxide sensors. While identifying $\mathrm{CH}_{4}, \mathrm{CO}$ and their gas mixtures, a test set classification accuracy rate of $98.67 \%$ was achieved, which was more accurate than ANNs and SVMs.

In [231], the aim was to identify the scents released from a landfill with respect to their odor nuisance. An EN system was designed with six semiconductor gas sensors by Figaro and its performance was compared with the commercially available Heracles II EN. The data samples were collected from different locations around the landfill and classified with LDA using cross-validation. Although Heracles gave better results, the developed prototype EN provided a classification success rate of $40 \%$.

The EN technology can further be employed to assess the air quality near municipal processing plants ${ }^{[50]}$. It is very important to monitor the surrounding area of these plants due to the odor nuisance. For instance, an EN network can be built to determine the unpleasant odors present around the plant. Data transmission can be actualized through the Internet, therefore the system can be remotely reached by operators. In locations where the environment cannot be held under control, it is important to employ robust ENs to avoid the interference of parameters, such as humidity, in the gas classification process $^{[18]}$.

\subsection{Security systems}

Because of their high selectivity, portability, wide linear range, minimal space and power requirements and low deployment cost in mass production, ENs are employed also in security applications ${ }^{232]}$. Moreover, this technology received tremendous attention from countries for national security in particular for detecting explosive compounds ${ }^{[233]}$. Similar to homeland security, ENs are further adopted in monitoring and protecting confined spaces. For example, an environment monitoring experiment was performed in [234] to analyze atmospheric air samples using an EN with metal-oxide sensors, along with oxygen, temperature and humidity sensors. The gas samples were stored in two distinct gas cylinders, in which one contains hydrogen sulphide, carbon monoxide and methane while the other contains air with zero grades. Experimental results illustrated the success of ENs in monitoring enclosed areas.

\subsection{Packaging}

ENs are useful devices in controlling the freshness of commercial products. In [235], an EN study (using 
PEN 2) was accomplished to analyze packaging and freshness decay parameters of minced beef stored in high-oxygen modified atmosphere at different temperature conditions. A system based on PCA and cluster analysis was successfully utilized to determine the correct time of spoilage. As a result, the meat lasted 9 days at $4.3^{\circ} \mathrm{C}, 3-4$ days at $8.1^{\circ} \mathrm{C}$ and 2 days at $15.5^{\circ} \mathrm{C}$ on average. The EN technology can also be used in reducing food waste which is a growing issue around environmental and economical angles ${ }^{[236]}$. The shelf-life of products can be extended by using smart (intelligent) packaging tools, which in return leads to a decrease in wasted food. Recent advances of intelligent packaging tools for freshness monitoring via optical (optoelectronic nose) systems can be found in [236].

\section{Challenges in EN systems}

Despite the fact that the EN technology develops rapidly with success, EN devices still contain hardware and software challenges, which need to be further eliminated. In this paper, major difficulties are investigated as below.

\subsection{Sensor sensitivity}

Sensor types such as MOS, QCM, BAW and SAW are frequently used in EN devices. Unfortunately, these sensors are sensitive to temperature and humidity changes, which indeed is a serious drawback of ENs especially to be used in food and beverage industries. After the production of mass spectrometry based ENs (which are expensive and complicated in operation), the sensitivity problem was solved ${ }^{[146]}$. Additionally, problems like sensor poisoning and the response signal nonlinearity were eliminated.

\subsection{Sensor selectivity}

Selectivity (the detection and measurement of desired chemicals) is noted as a difficulty in sensor arrays ${ }^{[20]}$. Hitherto, there has not been an accurate mathematical model developed to solve this issue. In [237], a method combining Deep Q Network (DQN) and CNNs was proposed to improve the selectivity of metal-oxide sensors. Multisensor sensing approaches ${ }^{[238,239]}$, fluctuation enhanced sensing ${ }^{[239,240]}$ and synchronizing the temperature in experiments are a few of the ideas put forward, but they do not provide a significant improvement for overcoming this challenge.

\subsection{Humidity}

ENs have several advantages compared to olfactometric methods, such as detecting and saving information about odor samples present in the environment for a very limited time ${ }^{[3]}$. However, gas arrays have to be calibrated according to olfactometric techniques in some fields where especially humidity is a challenge for ENs. Since poly- mers tend to absorb water vapor, the sensor sensitivity decreases when the humidity level in the environment increases ${ }^{[241]}$. As a result, this fact can cause the EN sensors to produce unreliable responses ${ }^{[242]}$ and odor samples with exactly the same characteristics may be classified as different gases.

In [243], a drying agent called anhydrous sodium carbonate was used to decrease the amount of water vapor for the detection of adulteration in cherry tomato juices. However, this employment reduced the detection accuracy. A successful strategy was proposed in [244] to overcome the humidity problem through a gas sensor array containing a nano-structured metal-oxides combined with metal-organic framework, with a significantly improved performance on sensor response and detection limit. $\mathrm{Hu}-$ midity remains a challenge in several practical EN applications such as development of mobile robots for gas source localization ${ }^{[25]}$, detection of respiratory diseases ${ }^{[246]}$ and monitoring environmental pollution ${ }^{[227]}$.

\subsection{Sensor drift}

Sensor drift, defined as the small changes in the output independent of the measured feature, is another issue in the EN technology. There are two reasons behind the drift problem. The first reason is the chemical process between the environment and sensor materials (first-order drift), and the second one is the noise produced in the system (second-order drift). Although various compensation techniques have been developed and the accuracy of drift driven gas recognition has greatly been enhanced, just a small number of these proposed methods consider online processing, e.g., [247]. Moreover, there are sensor selection strategies, long-lasting materials, post-processing mechanisms and transfer learning techniques to cancel out the drift.

\subsection{Sensor stability}

Over time, sensor drift tends to increase. For this reason, sensor response differs in time for the same odor. This causes a problem during gas classification with an algorithm ${ }^{248]}$. Baseline manipulation, through which the sensor output is transformed by using the initial response, is a common method to enhance sensor stability.

In particular, metal-oxide sensors have poor long-term stability and their employment in commercial applications is impractical due to this issue ${ }^{[249]}$. A successful study was conducted in [249] to decrease the drift and improve the stability of metal-oxide sensors. The proposed method was based on baseline manipulation alongside orthogonal signal correction and PLSR. In addition, nano-structured materials are one of the preferred paths to overcome the stability problem. Recently in [250], tinoxide nanosensors were proposed to improve sensor stability in ENs. While these nanosensors presented a good 
performance up to $100^{\circ} \mathrm{C}$, stability issues occurred at higher temperatures.

\subsection{Reproducibility}

Reproducibility is a common challenge in ENs. Signal characteristics of the chemical sensor response change in time because of repetitive usage ${ }^{[251]}$. Signal generation and interior variation in sensors occur due to the same environmental procedures, therefore this challenge cannot be entirely eliminated. However, it is possible to decrease the alteration degree by using durable materials in sensors. Another issue regarding reproducibility is related to the manufacturing stage of sensors. Since the sensing properties of produced sensors are only nearly identical, trying to replace a single sensor in an EN would result in reproducibility problems ${ }^{[252,253]}$. As a solution to this challenge, sensors in an array were divided into blocks in [254]. Whenever a sensor fails to produce a response, the block in which the failed sensor belongs to represents the missing sensor response through a linear model.

\subsection{Sensor fault tolerance}

During the sensor array design, it is a critical aspect to consider that a sensor failure can be unnoticeable due to poor design control[255]. Manufacturing defects, ageing and environmental conditions are some of the reasons for sensor failure, which usually results in a decrease in the classification accuracy. A system with a sensor array based on several clusters was proposed in [255] to tolerate sensor failure in artificial olfactory systems. The developed method achieved an odor classification success rate of $97.92 \%$ even though sensor failure was present in the system. In [256], an algorithm called self-repairing (SR) was designed to replace the broken sensors with replicas, without recalibrating the whole system or stopping the ongoing procedure. When compared to the standard classifiers, e.g., KNNs, LDA, PLSR, the SR algorithm presented better performance in increasing the fault tolerance.

\subsection{Cross sensitivity}

In practical applications, cross sensitivity can be defined as the interference of an unwanted smell to the odor that is desired to be detected ${ }^{[25]}$. Irrelevant substances are always present in the environment which can mislead the EN device and cause incorrect outcomes.

\subsection{Size of sensor array}

Yet another challenge is the large sizes of the sensor arrays ${ }^{[146]}$. To place ENs effectively in the industry and households, the size of the system has to be reduced.

\subsection{Material selection}

For practical applications, material selection in the design process of ENs is a key factor for achieving high success rates. To choose optimal polymers, analysis of sensor array response or linear solvation energy relationship parameters of polymers and analytes approaches can be used ${ }^{[258]}$. Moreover, the gas signatures obtained from sensors must be distinct in the feature space, otherwise the adopted algorithm will have difficulties in the classification stage ${ }^{[259]}$.

\subsection{Algorithms and parameter selection}

Importantly, the employed machine learning and pattern analysis algorithm(s) must meet the requirements of EN systems. It is not easy to choose the correct approach and sometimes none of the available methods is sufficient for the targeted task ${ }^{[260]}$. For instance, ENs are adopted in Chinese Herbal Medicines, however these are very complex and usually contain a varieties odors with different strengths ${ }^{[261]}$. Hence, the designed algorithm must be highly accurate in order to use EN systems in practice.

Hyper-parameter selection in machine learning algorithms is a very crucial step for developing robust and efficient systems. Grid search and manual search are two popular methods in choosing hyper-parameters. However, experimental results in [262] prove that random search is more efficient than these two search methods. Random search also provides freedom while performing experiments, i.e., an interruption during the process does not necessarily cause a requirement of the grid to be readjusted. Moreover, new computational resources can be easily introduced to ongoing experiments for additional trials without any need of reusing the same hyper-parameters.

\subsection{Lack of data}

Collecting sufficient and reliable data is usually a difficult, laborious and long process. For specific processing tasks in EN applications, unsupervised learning and clustering algorithms can be employed if the acquired data is limited ${ }^{[163]}$. To overcome the shortage of the labeled data, semi-supervised learning can be preferred ${ }^{[263]}$.

\section{Perspectives and future directions}

The understanding process of smell differs in many people as the subjective past experience changes the way they perceive things. Consequently, the standardization of odors is a complicated process which cannot be completely actualized by the odor experts ${ }^{[264]}$. However, with the combination of a proper sensor array and suitable ML methods, an EN system which behaves similar to the mammalian olfactory system can standardize aromas. 
After sensing the scents and processing these signals, the visualization of diverse aromas can be accomplished via wide range pattern recognition algorithms ${ }^{[265]}$.

Olfactory sensory neurons, which have one odor receptor in each, are present in human nose and they allow a connection from the nose to the brain ${ }^{[266]}$. The perceived odors are later classified in the brain and genes encoding receptor proteins help understand how the identification of odors occurs ${ }^{[267]}$. The distinct structures of these receptor proteins decide on the interaction with different substances ${ }^{265]}$. The reaction spectrum of various sensory neurons is established through the molecular receptive range of these receptors ${ }^{[268]}$. All findings achieved about these genes led to the development of bioelectronic noses. A bioelectronic nose contains two parts, the primary transducer which recognizes the biological data and the secondary transducer that transforms the biological data into a quantitative signal[269]. QCMs, field-effect transistors and conducting polymers are some of the materials employed as the secondary transducer. Furthermore, it is observed that nanomaterials are highly sensitive when they are used as secondary transducers. Therefore, tools such as nanomaterial based field-effect transistors and carbon nanotubes are adopted in bioelectronic noses.

There are two types of bioelectronic noses ${ }^{[270]}$. As the first type, protein-based biosensor exploits the olfactory receptor protein and it can directly detect the binding between odorant and receptor. The second kind is the cell-based biosensor and it employs living cells. Cell-based biosensors are considered to be economic and can be used for intrinsic signal measurement of cells. Moreover, through taking advantage of substances such as synthetic polypeptides, the properties of biosensors can be further developed ${ }^{[43]}$. In general, bioelectronic noses have the potential to be adopted as devices to detect and identify odorants at low concentration levels ${ }^{[265]}$. SAW sensors and odorant-binding proteins based bioelectronic noses have already been used to detect different molecules in food $^{[271]}$. In upcoming years, the detection of hazardous gases might be a field where bioelectronic noses could be employed.

Data fusion defined as combining the outputs of multiple sensors, databases and tools is a promising method to enhance EN systems ${ }^{[272]}$. Using ENs together with other devices such as e-tongues can improve their effectiveness. For instance, an EN and an e-tongue were adopted in [273] to detect the submerged fermentation of Tremella aurantialba, which is an edible jelly fungus. Fusion of the data from both tools led to encouraging results. Furthermore, an other data fusion system was designed in [274] to classify and assess edible olive oil quality. The data was gathered through three devices (using an EN, an etongue and an electronic eye) and the oil samples were successfully classified into two classes according to their freshness level with an average accuracy of $94 \%$. In con- clusion, data fusion is a promising method to further enhance the EN technology in the near future.

Lately, the concept of microfluidics has been introduced to gas sensing technologies. Microfluidics deals with systems that control and transport small amount of fluids in microscale dimensions ${ }^{[275]}$. Although some claim that microfluidic-based gas detectors can be an alternative to $\mathrm{ENs}^{[276]}$, the microfluidics concept may become a big step forward for ENs with the right combinations and modifications. As an example, a metal-oxide sensor was put together with an open digital microfluidic system in [277] for the classification of wine types. The developed system was successful at identifying different types of wine.

Chemometric tools are another promising technology for the EN development. Chemometrics is an interdisciplinary science of gathering data from chemical systems ${ }^{[278]}$ and has already been used in several EN applications ${ }^{[278,279]}$. Recently in [83], an EN containing 10 metaloxide sensors incorporated with chemometrics was employed to identify different stages of postharvest kiwifruit ripeness. LDA was adopted for classification with a crossvalidation accuracy of $99.4 \%$.

Flexible circuits have come on to the scene of circuit technology and can be utilized in wearable textiles ${ }^{[280]}$. By combining flexible circuits with ENs, health monitoring of astronauts can be achieved minutely in the upcoming space missions. Moreover, the brain computer interface (BCI) systems may be employed in the EN technology. The odors at remote distances can be sensed by an EN and a signal through an adopted BCI can be sent to the olfactory cortex, resulting in an artificial olfactory system $^{[281]}$. The combination of flexible circuits and BCI with the EN technology would definitely lead to more advanced systems.

Regarding algorithms, machine learning together with data analysis and pattern recognition tools need to demonstrate higher accuracy in actual environments after being trained with suitable datasets. For instance, in some practical applications such as $[145,184,207,210$, $212,261]$ the overall precision of the algorithms was lower than desired. Hitherto, deep learning adoption in the EN technology is rarely encountered in the literature, e.g.,

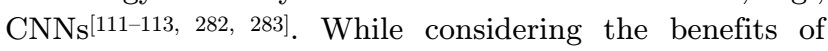
deep learning in other research domains, these tools should be used more frequently to increase the accuracy of ENs. In terms of multi-dimensional CNNs, the output of the sensor array can be converted into images to train and test the network. Crucially, this conversion process has to be relevant and robust, otherwise data loss and critical errors may occur. On the other side, there are also very rare studies reported in the literature which use onedimensional CNNs for mixture gases recognition and classification $^{[282,283]}$.

The nature of the EN problem in terms of machine learning algorithms would lead to a conclusion that 
sparsity-related tools could be very efficient. For the moment, to the best of available knowledge, just a small number of studies relate this problem in a sparse and redundant representations perspective. Some related research samples can be given but no more than in [284, 285]. By nature, the sparsity property strongly holds in most real world examples. As an example, interactions between proteins are very selective in molecular biology. Similarly, social network friendships are sparse, i.e., not everyone is a friend with everyone. A powerful example can be given as the working principles of the human brain. The brain, based on its functional segments, not only has spatial but also temporal sparsity. Just a small number of neurons are active in only very short time intervals ${ }^{[286]}$.

In more detail, natural smell-prints of each individual volatile organic compound should be sparse in nature, hence can be successfully distinguished from others via algorithms exploiting this natural phenomenon. In contrast to frequently used dimensionality reduction methods such as PCA and LDA, sparse learning algorithms apply an appropriate data transformation to sparsify the data representation, while increasing the space dimensionality. Later within this new higher-dimensional space, a search for related subspaces can be employed for classification or clustering purposes ${ }^{[286]}$. Another interesting aspect of the sparsity related concept is that structural constraints are highly possible to be introduced into the optimization problem. Group sparsity ${ }^{[287-290]}$ and its specific varieties, such as block sparsity ${ }^{[291,292]}$, are examples of additionally employed structural constraints into sparse learning algorithms, in which the support of sparse codes generally define distinct subspaces and later can be used for the classification problem, e.g., [293]. As a result, one possible algorithms related research can be in the direction of the structurally constrained sparsity concept for the mixture of gases detection and classification problem. Furthermore, related to block sparsity, deep sparse structures ${ }^{[286]}$ would be another encouraging aspect as a practical application to the EN technology paving the way for more successful EN devices. (Interested readers are recommended to refer to [294] for theoretical foundations and practical applications of sparse and redundant representations.)

Last but not least, the up-to-date practical trend in deep learning methodologies is of increasing the number and variety of training samples with different augmentation tools, and even more, with external data sources which apparently leads to much better success rates ${ }^{[295]}$. More crucially, the overall success rates are obviously improved with ensembles of several classifiers and output fusion techniques. Ensembles of deep learning techniques with supporting techniques such as widely used SVMs and even sparse models could be another alternative promising research direction in the EN technology.

\section{Conclusions}

Transferring human characteristics to non-living systems takes us one step closer to the science fiction stories in our dreams. The replication of human senses has been one of the most popular research topics for decades and the EN technology is a product of these long term studies. The human olfactory system is extremely complex, however it is not impossible to replicate. Since the day of the first prototype EN was developed, it has become a useful device in several applications, in solving urgent problems. Especially in the food industry and medicine, traditional ways of distinguishing subjects are too slow, expensive and mostly subjective which may result in fatal errors. The EN technology instead offers a fast, sensitive, low cost and objective alternative. Moreover, the potential of this new way of sensing is still rapidly growing with the new developments in sensors and machine learning technologies.

An EN consists of two main components, the sensor array which is responsible for sensing the chemicals and algorithms which provide an analyzing software model in the system. There are several improvements required in both parts. More compact sensors need to be designed with the rising importance of wearable technologies. In the near future, the production of robust nanosensors for various tasks will presumably eliminate this difficulty. A further concern in ENs is the recalibration of sensors. Particularly, dynamic systems such as metal-oxide gas sensors require regular recalibration ${ }^{[296]}$, which leads to time waste and high economic costs. Manufacturing stable sensors with durable materials will save time and money, especially in space missions, medicine and security applications.

Moreover, algorithms need to be fast and accurate in satisfying the requirements of the system for the targeted task. Another major issue remaining to be solved is the low number of publicly available research datasets. In almost every individual study, a setup has been designed to form a dataset ${ }^{[296]}$, which is indeed time consuming. On the other hand, the problem naturally requires specific datasets to be collected due to the used hardware characteristics, i.e., types and numbers of sensors may be different from one application to another. If these already collected datasets for chemical classification can be made publicly available in a common space, not only a great amount of time will be saved but also further competing research can be carried out on these datasets. Additionally, reducing or eliminating the noise from collected data will sharply increase the accuracy of EN systems.

Smell does not contain features like image textures, colors and edges. Therefore, creating a neural network structure based on the olfaction system results in lower computational time compared to that of computer vision [297]. Much less data is required to train the system with relatively efficient outcomes to test for. In experi- 
ments which the computational time is limited and rough experimental results are acceptable, olfaction-inspired neural networks can be preferred. Hence, olfaction-inspired neural networks hold a development-potential for the software part of the EN technology.

As a final note, a new practical application of the EN technology might be the identification of acute necrotizing ulcerative gingivitis (ANUG), which is a disease arising on the papillas of the gingiva. ANUG is usually a result of stress and it is mostly seen among old and middle aged women. It causes the papillas to turn white in color and a bad smell emerges inside the mouth. The odor released is sometimes confused with other diseases and a reliable solution for this issue is not present at the moment. Therefore, an EN will be helpful for dentists to identify ANUG.

\section{Acknowledgements}

The authors are thankful to Betul Arslan, Dentist, for fruitful discussions about dental health-care, which lead to innovative ideas.

\section{Open Access}

This article is distributed under the terms of the Creative Commons Attribution 4.0 International License (http://creativecommons.org/licenses/by/4.0/), which permits unrestricted use, distribution, and reproduction in any medium, provided you give appropriate credit to the original author(s) and the source, provide a link to the Creative Commons license, and indicate if changes were made.

\section{References}

[1] T. M. Dymerski, T. M. Chmiel, W. Wardencki. Invited review article: An odor-sensing system-powerful technique for foodstuff studies. Review of Scientific Instruments, vol.82, no.11, Article number 111101, 2011. DOI: $10.1063 / 1.3660805$.

[2] K. Mori. The Olfactory System: From Odor Molecules to Motivational Behaviors, Tokyo, Japan: Springer, 2014. DOI: $10.1007 / 978-4-431-54376-3$.

[3] B. Szulczynski, K. Arminski, J. Namiesnik, J. Gebicki. Determination of odour interactions in gaseous mixtures using electronic nose methods with artificial neural networks. Sensors, vol.18, no. 2, Article number 519, 2018. DOI: $10.3390 / \mathrm{s} 18020519$.

[4] H. Zwaardemaker, F. Hogewind. On spray-electricity and waterfall electricity. In Proceedings of the Royal Netherlands Academy of Arts and Sciences, KNAW, Amsterdam, The Netherlands, pp.429-437, 1920.

[5] B. Berglund, U. Berglund, T. Lindvall, L. T. Svensson. A quantitative principle of perceived intensity summation in odor mixtures. Journal of Experimental Psychology, vol. 100, no. 1, pp. 29-38, 1973. DOI: 10.1037/h0035435.

[6] P. Laffort, A. Dravnieks. Several models of suprathreshold quantitative olfactory interaction in humans ap- plied to binary, ternary and quaternary mixtures. Chemical Senses, vol.7, no. 2, pp.153-174, 1982. DOI: 10. 1093/chemse/7.2.153.

[7] P. Laffort. Models for describing intensity interactions in odor mixtures: A reappraisal. Perception of Complex Smells and Tastes, D. G. Laing, W. S. Cam, R. L. McBride, B. W. Ache, Eds., New York, USA: Academic Press, pp. 20-223, 1989.

[8] P. Laffort. The application of synergy and inhibition phenomena to odor reduction. Studies in Environmental Science, vol.61, pp.105-117, 1994. DOI: 10.1016/S01661116(08)72047-8.

[9] B. Szulczynski, J. Namiesnik, J. Gebicki. Determination of odour interactions of three-component gas mixtures using an electronic nose. Sensors, vol.17, no.10, Article number 2380, 2017. DOI: 10.3390/s17102380.

[10] L. C. Yan, J. M. Liu, S. Jiang, C. D. Wu, K. W. Gao. The regular interaction pattern among odorants of the same type and its application in odor intensity assessment. Sensors, vol.17, no.7, Article number 1624, 2017. DOI: $10.3390 / \mathrm{s} 17071624$.

[11] G. Hudon, C. Guy, J. Hermia. Measurement of odor intensity by an electronic nose. Journal of the Air \& Waste Management Association, vol.50, no.10, pp.1750-1758, 2000. DOI: 10.1080/10473289.2000.10464202.

[12] K. Persaud, G. Dodd. Analysis of discrimination mechanisms in the mammalian olfactory system using a model nose. Nature, vol.299, no. 5881, pp.352-355, 1982. DOI: $10.1038 / 299352 \mathrm{a} 0$.

[13] A. Ikegami, M. Kaneyasu. Olfactory detection using integrated sensors. In Proceedings of the 3rd International Conference on Solid-State Sensors and Actuators and Microsystems, IEEE, Philadelphia, USA, pp. 136-139, 1985.

[14] M. A. Craven, J. W. Gardner, P. N. Bartlett. Electronic noses-development and future prospects. TrAC Trends in Analytical Chemistry, vol.15, no.9, pp.486-493, 1996. DOI: 10.1016/S0165-9936(96)00061-1.

[15] T. Pobkrut, T. Eamsa-ard, T. Kerdcharoen. Sensor drone for aerial odor mapping for agriculture and security services. In Proceedings of the 13th International Conference on Electrical Engineering/Electronics, Computer, Telecommunications and Information Technology, IEEE, Chiang Mai, Thailand, 2016. DOI: 10.1109/ECTICon. 2016.7561340.

[16] A. Rocha, M. Serrhini, C. Felgueiras. Europe and MENA Cooperation Advances in Information and Communication Technologies, Cham, Germany: Springer, 2017.

[17] M. J. Friedrich. Scientists seek to sniff out diseases. $J A M A$, vol.301, no.6, pp.585-586, 2009. DOI: 10.1001/ jama.2009.90.

[18] A. D. Wilson, M. Baietto. Applications and advances in electronic-nose technologies. Sensors, vol.9, no. 7, pp. 5099-5148, 2009. DOI: 10.3390/s90705099.

[19] K. Arshak, E. Moore, G. M. Lyons, J. Harris, S. Clifford. A review of gas sensors employed in electronic nose applications. Sensor Review, vol.24, no. 2, pp.181-198, 2004. DOI: 10.1108/02602280410525977.

[20] A. Loutfi, S. Coradeschi, G. K. Mani, P. Shankar, J. B. B. Rayappan. Electronic noses for food quality: A review. Journal of Food Engineering, vol. 144, pp.103-111, 2015. DOI: 10.1016/j.jfoodeng.2014.07.019. 
[21] B. Thakral, S. Gupta. Artificial neural networks for eNOSE: A review. International Journal of Applied Engineering Research, vol. 7, no. 11, pp. 1601-1604, 2012.

[22] R. Pallas-Areny, J. G. Webster. Sensors and Signal Conditioning, 2nd ed., New York, USA: Wiley, 2000.

[23] C. Sarafoleanu, C. Mella, M. Georgescu, C. Perederco. The importance of the olfactory sense in the human behavior and evolution. Journal of Medicine and Life, vol.2, no. 2, pp. 196-198, 2009.

[24] B. Szulczynski, J. Gebicki. Currently commercially available chemical sensors employed for detection of volatile organic compounds in outdoor and indoor air. Environments, vol.4, no.1, Article number 21, 2017. DOI: 10.3390/environments4010021.

[25] P. Boeker. On 'electronic nose' methodology. Sensors and Actuators B: Chemical, vol.204, pp.2-17, 2014. DOI: 10.1016/j.snb.2014.07.087.

[26] J. Gebicki. Application of electrochemical sensors and sensor matrixes for measurement of odorous chemical compounds. TrAC Trends in Analytical Chemistry, vol. 77, pp.1-13, 2016. DOI: 10.1016/j.trac.2015.10.005.

[27] C. X. Wang, L. W. Yin, L. Y. Zhang, D. Xiang, R. Gao. Metal oxide gas sensors: Sensitivity and influencing factors. Sensors, vol.10, no.3, pp. 2088-2106, 2010. DOI: $10.3390 / \mathrm{s} 100302088$.

[28] A. Berna. Metal oxide sensors for electronic noses and their application to food analysis. Sensors, vol.10, no. 4, pp. 3882-3910, 2010. DOI: 10.3390/s100403882.

[29] N. Yamazoe, Y. Kurokawa, T. Seiyama. Effects of additives on semiconductor gas sensors. Sensors and Actuators, vol.4, pp.283-289, 1983. DOI: 10.1016/0250$6874(83) 85034-3$.

[30] H. J. Kim, J. H. Lee. Highly sensitive and selective gas sensors using p-type oxide semiconductors: Overview. Sensors and Actuators B: Chemical, vol. 192, pp.607-627, 2014. DOI: $10.1016 /$ j.snb.2013.11.005.

[31] H. Bai, G. Q. Shi. Gas sensors based on conducting polymers. Sensors, vol.7, no.3, pp.267-307, 2007. DOI: $10.3390 / \mathrm{s} 7030267$.

[32] X. H. Huang, Q. S. Bai, J. G. Hu, D. Hou. A practical model of quartz crystal microbalance in actual applications. Sensors, vol. 17, no. 8, Article number 1785, 2017. DOI: $10.3390 / \mathrm{s} 17081785$.

[33] N. W. Turner, M. Bloxham, S. A. Piletsky, M. J. Whitcombe, I. Chianella. The use of a quartz crystal microbalance as an analytical tool to monitor particle/surface and particle/particle interactions under dry ambient and pressurized conditions: A study using common inhaler components. Analyst, vol.142, no. 1, pp.229-236, 2017. DOI: $10.1039 /$ C6AN01572G.

[34] J. W. Thies, P. Kuhn, B. Thurmann, S. Dubel, A. Dietzel. Microfluidic quartz-crystal-microbalance (QCM) sensors with specialized immunoassays for extended measurement range and improved reusability. Microelectronic Engineering, vol.179, pp.25-30, 2017. DOI: 10.1016/j.mee.2017.04.023.

[35] Y. Yan, D. D. Lu, H. Hao, H. P. Hou, T. Zhang, L. M. $\mathrm{Wu}$, L. K. Cai. Polyaniline-modified quartz crystal microbalance sensor for detection of formic acid gas. Water, Air, \& Soil Pollution, vol.223, no.3, pp. 1275-1280, 2012. DOI: $10.1007 / \mathrm{s} 11270-011-0943-1$.

[36] A. Alassi, M. Benammar, D. Brett. Quartz crystal mi- crobalance electronic interfacing systems: A review. Sensors, vol.17, no.12, Article number 2799, 2017. DOI: $10.3390 / \mathrm{s} 17122799$.

[37] S. K. Jha, K. Hayashi. A quick responding quartz crystal microbalance sensor array based on molecular imprinted polyacrylic acids coating for selective identification of aldehydes in body odor. Talanta, vol.134, pp.105-119, 2015. DOI: 10.1016/j.talanta.2014.09.049.

[38] T. Wasilewski, B. Szulczynski, M. Wojciechowski, W. Kamysz, J. Gebicki. A highly selective biosensor based on peptide directly derived from the HarmOBP7 aldehyde binding site. Sensors, vol.19, no. 19, Article number 4284, 2019. DOI: $10.3390 / \mathrm{s} 19194284$.

[39] S. Sankaran, S. Panigrahi, S. Mallik. Olfactory receptor based piezoelectric biosensors for detection of alcohols related to food safety applications. Sensors and Actuators $B$ : Chemical, vol. 155, no. 1, pp.8-18, 2011. DOI: 10.1016/j.snb.2010.08.003.

[40] Y. S. Zhang, K. Yu, R. L. Xu, D. S. Jiang, L. Q. Luo, Z. Q. Zhu. Quartz crystal microbalance coated with carbon nanotube films used as humidity sensor. Sensors and Actuators A: Physical, vol.120, no.1, pp.142-146, 2005. DOI: $10.1016 /$ j.sna.2004.11.032.

[41] P. J. Qi, C. Zhao, R. Wang, T. Fei, T. Zhang. High-performance QCM humidity sensors using acidized-multiwalled carbon nanotubes as sensing film. IEEE Sensors Journal, vol. 18 , no. 13 , pp. $5278-5283$, 2018. DOI: 10.1109/JSEN.2018.2839110.

[42] I. A. Koshets, Z. I. Kazantseva, Y. M. Shirshov, S. A. Cherenok, V. I. Kalchenko. Calixarene films as sensitive coatings for QCM-based gas sensors. Sensors and Actuators B: Chemical, vol.106, no.1, pp.177-181, 2005. DOI: 10.1016/j.snb.2004.05.054.

[43] T. Wasilewski, B. Szulczynski, W. Kamysz, J. Gebicki, J. Namiesnik. Evaluation of three peptide immobilization techniques on a QCM surface related to acetaldehyde responses in the gas phase. Sensors, vol.18, no.11, Article number 3942, 2018. DOI: 10.3390/s18113942.

[44] G. Sauerbrey. Verwendung von schwingquarzen zur wagung dunner schichten und zur mikrowagung. Zeitschrift fur Physik, vol. 155, no. 2, pp. 206-222, 1959. (in German) DOI: $10.1007 / \mathrm{BF} 01337937$.

[45] J. D. N. Cheeke, Z. Wang. Acoustic wave gas sensors. Acoustic wave gas sensors. Sensors and Actuators B: Chemical, vol.59, no.2-3, pp.146-153, 1999. DOI: 10.1016/S0925-4005(99)00212-9.

[46] J. W. Gardner, P. N. Bartlett. Electronic Noses: Principles and Applications, Oxford, UK: Oxford University Press, 1999.

[47] X. Liu, S. T. Cheng, H. Liu, S. Hu, D. Q. Zhang, H. S. Ning. A survey on gas sensing technology. Sensors, vol. 12, no. 7, pp.9635-9665, 2012. DOI: 10.3390/ s120709635.

[48] S. Esfahani, J. A. Covington. Low cost optical electronic nose for biomedical applications. Proceedings, vol.1, no. 4, Article number 589, 2017. DOI: 10.3390/proceedings1040589.

[49] L. Spinelle, M. Gerboles, G. Kok, S. Persijn, T. Sauerwald. Review of portable and low-cost sensors for the ambient air monitoring of benzene and other volatile organic compounds. Sensors, vol.17, no.7, Article number 1520, 2017. DOI: $10.3390 / \mathrm{s} 17071520$. 
[50] B. Szulczynski, T. Wasilewski, W. Wojnowski, T. Majchrzak, T. Dymerski, J. Namiesnik, J. Gebicki. Different ways to apply a measurement instrument of e-nose type to evaluate ambient air quality with respect to odour nuisance in a vicinity of municipal processing plants. Sensors, vol.17, no.11, Article number 2671, 2017. DOI: $10.3390 / \mathrm{s} 17112671$.

[51] I. El Naqa, M. J. Murphy. What is machine learning? Machine Learning in Radiation Oncology: Theory and Applications, I. El Naqa, R. J. Li, M. J. Murphy, Eds., Cham, Germany: Springer, pp.3-11, 2015. DOI: 10. 1007/978-3-319-18305-3_1.

[52] G. Wiederhold, J. McCarthy. Arthur Samuel: Pioneer in machine learning. IBM Journal of Research and Development, vol.36, no.3, pp.329-331, 1992. DOI: 10.1147/ rd.363.0329.

[53] E. Alpaydin. Introduction to Machine Learning, Cambridge, UK: MIT Press, 2014.

[54] O. Chapelle, B. Scholkopf, A. Zien. Semi-supervised Learning, Cambridge, UK: MIT Press, 2010.

[55] A. D. Wilson. Review of electronic-nose technologies and algorithms to detect hazardous chemicals in the environment. Procedia Technology, vol.1, pp.453-463, 2012. DOI: $10.1016 / \mathrm{j}$.protcy.2012.02.101.

[56] C. M. Bishop. Neural Networks for Pattern Recognition, New York, USA: Oxford University Press, 1995.

[57] C. M. Bishop. Pattern Recognition and Machine Learning, New York, USA: Springer, 2013.

[58] S. Al Maskari, X. Li. E-nose pattern recognition and drift compensation methods. Electronic Nose Technologies and Advances in Machine Olfaction, Y. A. Albastaki, F. Albalooshi, Eds., Hershey, USA: Engineering Science Reference, pp.38-57, 2018. DOI: 10.4018/978-1-5225-38622.ch003.

[59] G. J. McLachlan. Discriminant Analysis and Statistical Pattern Recognition, New York: USA, Wiley, 2005.

[60] R. O. Duda, P. E. Hart, D. G. Stork. Pattern Classification, 2nd ed., New York: USA: John Wiley and Sons, 2000.

[61] X. J. Tian, J. Wang, S. Q. Cui. Analysis of pork adulteration in minced mutton using electronic nose of metal oxide sensors. Journal of Food Engineering, vol.119, no. 4, pp. 744-749, 2013. DOI: 10.1016/j.jfoodeng.2013.07.004.

[62] V. E. Vinzi, W. W. Chin, J. Henseler, H. W. Wang. Handbook of Partial Least Squares: Concepts, Methods and Applications, New York, USA: Springer, 2010.

[63] T. Aguilera, J. Lozano, J. A. Paredes, F. J. Alvarez, J. I. Suarez. Electronic nose based on independent component analysis combined with partial least squares and artificial neural networks for wine prediction. Sensors, vol. 12, no. 6, pp. 8055-8072, 2012. DOI: 10.3390/s120608055.

[64] T. Amemiya. Advanced Econometrics. Harvard University Press, Cambridge, USA, 1985.

[65] P. Giungato, E. Laiola, V. Nicolardi. Evaluation of industrial roasting degree of coffee beans by using an electronic nose and a stepwise backward selection of predictors. Food Analytical Methods, vol.10, no.10, pp.3424-3433, 2017. DOI: $10.1007 / \mathrm{s} 12161-017-0909-\mathrm{z}$.

[66] C. Baskar, N. Nesakumar, J. B. B. Rayappan, M. Doraipandian. A framework for analysing E-nose data based on fuzzy set multiple linear regression: Paddy quality as- sessment. Sensors and Actuators A: Physical, vol.267, pp. 200-209, 2017. DOI: 10.1016/j.sna.2017.10.020.

[67] I. Jolliffe. Principal component analysis. International Encyclopedia of Statistical Science, M. Lovric, Ed., Berlin, Germany: Springer, 2011. DOI: 10.1007/978-3-64204898-2_455.

[68] L. P. Wang. Support Vector Machines: Theory and Applications, Berlin, Germany: Springer, 2005.

[69] R. Laref, E. Losson, A. Sava, K. Adjallah, M. Siadat. A comparison between SVM and PLS for E-nose based gas concentration monitoring. In Proceedings of IEEE International Conference on Industrial Technology, IEEE, Lyon, France, pp. 1335-1339, 2018. DOI: 10.1109/ICIT. 2018.8352372 .

[70] J. M. Zurada. Introduction to Artificial Neural Systems. St. Paul, USA: West Publishing House, 1992.

[71] Y. Thazin, T. Pobkrut, T. Kerdcharoen. Prediction of acidity levels of fresh roasted coffees using e-nose and artificial neural network. In Proceedings of the 10th International Conference on Knowledge and Smart Technology, IEEE, Chiang Mai, Thailand, pp.210-215, 2018. DOI: 10.1109/KST.2018.8426206.

[72] L. Zhang, F. C. Tian. Performance study of multilayer perceptrons in a low-cost electronic nose. IEEE Transactions on Instrumentation and Measurement, vol. 63, no. 7, pp. 1670-1679, 2014. DOI: 10.1109/TIM.2014.2298691.

[73] J. P. Santos, M. Garcia, M. Aleixandre, M. C. Horrillo, J. Gutierrez, I. Sayago, M. J. Fernandez, L. Ares. Electronic nose for the identification of pig feeding and ripening time in Iberian hams. Meat Science, vol.66, no.3, pp. 727-732, 2004. DOI: 10.1016/j.meatsci.2003.07.005.

[74] S. Romani, C. Cevoli, A. Fabbri, L. Alessandrini, M. Dalla Rosa. Evaluation of coffee roasting degree by using electronic nose and artificial neural network for off-line quality control. Journal of Food Science, vol.77, no.9, pp. C960-C965, 2012. DOI: 10.1111/j.1750-3841.2012. 02851.x.

[75] M. E. Escuderos, S. Sanchez, A. Jimenez. Quartz crystal microbalance (QCM) sensor arrays selection for olive oil sensory evaluation. Food Chemistry, vol.124, no.3, pp. 857-862, 2011. DOI: 10.1016/j.foodchem.2010.07.007.

[76] T. Saidi, K. Tahri, N. El Bari, R. Ionescu, B. Bouchikhi. Detection of seasonal allergic rhinitis from exhaled breath VOCs using an electronic nose based on an array of chemical sensors. In Proceedings of IEEE Sensors, IEEE, Busan, South Korea, 2015, DOI: 10.1109/ICSENS. 2015.7370579.

[77] P. Maho, C. Dolcinotti, T. Livache, C. Herrier, A. Andreev, P. Comon, Simon Barthelme. Olfactive robot for gas discrimination over several months using a new optoelectronic nose. In Proceedings of the 18th International Symposium on Olfaction and Electronic Nose, Fukuoka, Japan, 2019.

[78] T. Dymerski, J. Gebicki, W. Wardencki, J. Namiesnik. Quality evaluation of agricultural distillates using an electronic nose. Sensors, vol.13, no.12, pp.15954-15967, 2013. DOI: $10.3390 / \mathrm{s} 131215954$.

[79] S. Buratti, S. Benedetti, M. Scampicchio, E. C. Pangerod. Characterization and classification of Italian barbera wines by using an electronic nose and an amperometric electronic tongue. Analytica Chimica Acta, vol.525, no. 1, pp. 133-139, 2004. DOI: 10.1016/j.aca.2004.07.062. 
[80] V. Y. Musatov, V. V. Sysoev, M. Sommer, I. Kiselev. Assessment of meat freshness with metal oxide sensor microarray electronic nose: A practical approach. Sensors and Actuators B: Chemical, vol.144, no.1, pp.99-103, 2010. DOI: $10.1016 /$ j.snb.2009.10.040.

[81] X. Z. Hong, J. Wang. Discrimination and prediction of pork freshness by E-nose. In Proceedings of the 5th International Conference on Computer and Computing Technologies in Agriculture $V$, Springer, Beijing, China, pp. 1-14, 2011. DOI: 10.1007/978-3-642-27275-2_1.

[82] Z. B. Wei, X. Z. Xiao, J. Wang, H. Wang. Identification of the rice wines with different marked ages by electronic nose coupled with smartphone and cloud storage platform. Sensors, vol. 17, no. 11, Article number 2500, 2017. DOI: $10.3390 / \mathrm{s} 17112500$.

[83] D. D. Du, J. Wang, B. Wang, L. Y. Zhu, X. Z. Hong. Ripeness prediction of postharvest kiwifruit using a MOS E-nose combined with chemometrics. Sensors, vol.19, no. 2, Article number 419, 2019. DOI: 10.3390/s19020419.

[84] T. Dymerski, J. Gebicki, W. Wardencki, J. Namiesnik. Application of an electronic nose instrument to fast classification of polish honey types. Sensors, vol.14, no. 6 , pp. 10709-10724, 2014. DOI: 10.3390/s140610709.

[85] C. J. C. Burges. A tutorial on support vector machines for pattern recognition. Data Mining and Knowledge Discovery, vol.2, no. 2, pp.121-167, 1998. DOI: 10.1023/A: 1009715923555 .

[86] M. Goudjil, M. Koudil, M. Bedda, N. Ghoggali. A novel active learning method using SVM for text classification. International Journal of Automation and Computing, vol. 15, no. 3, pp. 290-298, 2018. DOI: 10.1007/s11633015-0912-z.

[87] P. Saha, S. Ghorai, B. Tudu, R. Bandyopadhyay, N. Bhattacharyya. Multi-class support vector machine for quality estimation of black tea using electronic nose. In Proceedings of the 6th International Conference on Sensing Technology, IEEE, Kolkata, India, pp. 571-576, 2012. DOI: 10.1109/ICSensT.2012.6461744.

[88] Z. Y. Chang, J. H. Lv, H. Y. Qi, Y. H. Ma, D. H. Chen, J. Xie, Y. H. Sun. Bacterial infection potato tuber soft rot disease detection based on electronic nose. Open Life Sciences, vol.12, no. 1, pp.379-385, 2017. DOI: 10.1515/biol2017-0044.

[89] R. V. Leal, A. X. C. Quiming, J. F. Villaverde, A. N. Yumang, N. B. Linsangan, M. V. C. Caya. Determination of schizophrenia using electronic nose via support vector machine. In Proceedings of the 9th International Conference on Biomedical Engineering and Technology, ACM, Tokyo, Japan, pp.13-17, 2019. DOI: 10.1145/3326172. 3326212 .

[90] A. K. Jain, J. C. Mao, K. M. Mohiuddin. Artificial neural networks: A tutorial. Computer, vol.29, no.3, pp.31-44, 1996. DOI: 10.1109/2.485891.

[91] M. L. R. Mendez. Electronic Noses and Tongues in Food Science, London, UK: Academic Press, 2016.

[92] K. Faceli, A. C. P. L. F. De Carvalho, S. O. Rezende. Combining intelligent techniques for sensor fusion. Applied Intelligence, vol.20, no. 3, pp.199-213, 2004. DOI: 10.1023/B:APIN.0000021413.05467.20.

[93] A. D. Wilson. Recent applications of electronic-nose technologies for the noninvasive early diagnosis of gastrointestinal diseases. Multidisciplinary Digital Publishing Institute Proceedings, vol. 2, no. 3, Article number
147, 2018. DOI: 10.3390/ecsa-4-04918.

[94] J. Chilo, J. Pelegri-Sebastia, M. Cupane, T. Sogorb. Enose application to food industry production. IEEE Instrumentation \& Measurement Magazine, vol.19, no.1, pp. 27-33, 2016. DOI: 10.1109/MIM.2016.7384957.

[95] Y. LeCun, Y. Bengio, G. Hinton. Deep learning. Nature, vol.521, no. 7553 , pp. 436-444, 2015. DOI: $10.1038 /$ nature 14539

[96] T. H. Chan, K. Jia, S. H. Gao, J. W. Lu, Z. N. Zeng, Y. Ma. PCANet: A simple deep learning baseline for image classification? IEEE Transactions on Image Processing, vol. 24, no. 12 , pp. 5017-5032, 2015. DOI: 10.1109/TIP. 2015.2475625 .

[97] K. M. He, X. Y. Zhang, S. Q. Ren, J. Sun. Deep residual learning for image recognition. In Proceedings of Conference on Computer Vision and Pattern Recognition, IEEE, Las Vegas, USA, pp. 770-778, 2016.

[98] B. Zhao, J. S. Feng, X. Wu, S. C. Yan. A survey on deep learning-based fine-grained object classification and semantic segmentation. International Journal of Automation and Computing, vol.14, no.2, pp.119-135, 2017. DOI: $10.1007 / \mathrm{s} 11633-017-1053-3$.

[99] A. X. Li, K. X. Zhang, L. W. Wang. Zero-shot finegrained classification by deep feature learning with semantics. International Journal of Automation and Computing, vol.16, no.5, pp.563-574, 2019. DOI: $10.1007 /$ s11633-019-1177-8.

[100] T. S. Buda, B. Caglayan, H. Assem. DeepAD: A generic framework based on deep learning for time series anomaly detection. Proceedings of the 22nd Pacific-Asia Conference Advances in Knowledge Discovery and Data Mining, Springer, Melbourne, Australia, pp.577-588, 2018. DOI: $10.1007 / 978-3-319-93034-3 \_46$.

[101] G. Litjens, T. Kooi, B. E. Bejnordi, A. A. A. Setio, F. Ciompi, M. Ghafoorian, J. A. W. M. van der Laak, B. van Ginneken, C. I. Sánchez. A survey on deep learning in medical image analysis. Medical Image Analysis, vol.42, pp.6-88, 2017. DOI: 10.1016/j.media.2017.07.005.

[102] L. C. Chen, G. Papandreou, I. Kokkinos, K. P. Murphy, A. L. Yuille. DeepLab: Semantic image segmentation with deep convolutional nets, atrous convolution, and fully connected CRFs. IEEE Transactions on Pattern Analysis and Machine Intelligence, vol.40, no.4, pp. 834-848, 2018. DOI: 10.1109/TPAMI.2017.2699184.

[103] Z. J. Yao, J. Bi, Y. X. Chen. Applying deep learning to individual and community health monitoring data: A survey. International Journal of Automation and Computing, vol. 15 , no. 6 , pp.643-655, 2018. DOI: $10.1007 /$ s11633-018-1136-9.

[104] C. Y. Wu, P. Karanasou, M. J. F. Gales, K. C. Sim. Stimulated deep neural network for speech recognition. In Interspeech 2016, International Speech Communication Association, San Francisco, USA, pp.400-404, 2016. DOI: 10.21437/Interspeech.2016-580.

[105] X. D. Sun, P. C. Wu, S. C. H. Hoi. Face detection using deep learning: An improved faster RCNN approach. Neurocomputing, vol. 299, pp.42-50, 2018. DOI: 10.1016/ j.neucom.2018.03.030.

[106] R. Ranjan, S. Sankaranarayanan, A. Bansal, N. Bodla, J. C. Chen, V. M. Patel, C. D. Castillo, R. Chellappa. Deep learning for understanding faces: Machines may be just as good, or better, than humans. IEEE Signal Processing Magazine, vol.35, no.1, pp.66-83, 2018. DOI: 10.1109/ 
MSP.2017.2764116.

[107] Y. Z. Lu. A novel face recognition algorithm for distinguishing faces with various angles. International Journal of Automation and Computing, vol. 5, no. 2, pp.193-197, 2008. DOI: 10.1007/s11633-008-0193-x.

[108] J. H. Tao, J. Huang, Y. Li, Z. Lian, M. Y. Niu. Semi-supervised ladder networks for speech emotion recognition. International Journal of Automation and Computing, vol.16, no.4, pp.437-448, 2019. DOI: 10.1007/s11633019-1175-x.

[109] S. Khan, H. Rahmani, S. A. A. Shah, M. Bennamoun, G. Medioni, S. Dickinson. A Guide to Convolutional Neural Networks for Computer Vision, Morgan \& Claypool Publishers, San Rafael, USA, pp.1-207, 2018. DOI: 10.2200/S00822ED1V01Y201712COV015.

[110] D. H. Hubel, T. N. Wiesel. Receptive fields, binocular interaction and functional architecture in the cat's visual cortex. The Journal of Physiology, vol.160, no.1, pp. 106-154, 1962. DOI: 10.1113/jphysiol.1962.sp006837.

[111] P. F. Qi, Q. H. Meng, M. Zeng. A CNN-based simplified data processing method for electronic noses. In Proceedings of ISOCS/IEEE International Symposium on Olfaction and Electronic Nose, IEEE, Montreal, Canada, pp. 1-3, 2017. DOI: 10.1109/ISOEN.2017.7968887.

[112] P. Peng, X. J. Zhao, X. F. Pan, W. B. Ye. Gas classification using deep convolutional neural networks. Sensors, vol.18, no. 1, Article number 157, 2018. DOI: $10.3390 / \mathrm{s} 18010157$.

[113] G. F. Wei, G. Li, J. Zhao, A. X. He. Development of a LeNet-5 gas identification CNN structure for electronic noses. Sensors, vol.19, no. 1, Article number 217, 2019. DOI: $10.3390 / \mathrm{s} 19010217$.

[114] H. E. Zhang, W. B. Ye, X. J. Zhao, R. K. F. Teng, X. F. Pan. A novel convolutional recurrent neural network based algorithm for fast gas recognition in electronic nose system. In Proceedings of IEEE International Conference on Electron Devices and Solid State Circuits, IEEE, Shenzhen, China, 2018. DOI: 10.1109/EDSSC.2018. 8487105 .

[115] D. H. Ackley, G. E. Hinton, T. J. Sejnowski. A learning algorithm for boltzmann machines. Cognitive Science, vol.9, no. 1, pp. 147-169, 1985. DOI: 10.1016/S0364-0213 (85)80012-4.

[116] R. Salakhutdinov, G. Hinton. Deep boltzmann machines. In Proceedings of the 20th International Conference on Artificial Intelligence and Statistics, Florida, USA, pp. 448-455, 2009.

[117] C. T. Tang, C. M. Huang, K. T. Tang, H. Chen. A scalable and adaptable probabilistic model embedded in an electronic nose for intelligent sensor fusion. In Proceedings of IEEE Biomedical Circuits and Systems Conference, IEEE, Atlanta, USA, 2015. DOI: 10.1109/BioCAS.2015.7348377.

[118] R. Kohavi, F. Provost. Glossary of terms. Glossary of terms. Machine Learning, vol.30, no.2-3, pp.271-274, 1998. DOI: $10.1023 / \mathrm{A}: 1017181826899$.

[119] Electronic Sensor Technology, Inc, CA, USA; Available: https://www.estcal.com/, October 21, 2019.

[120] AIRSENSE Analytics, Schwerin, Germany; Available: https://airsense.com/en, October 21, 2019.

[121] Electronic Nose Co., LTD, Bangkok, Thailand; Avail- able: http://e-nose.asia/index.php?lang=en, October 21, 2019.

[122] Sensigent, California, USA; Available: https://www. sensigent.com, October 21, 2019.

[123] FOODsniffer, California, USA; Available: http://www. myfoodsniffer.com/, October 21, 2019.

[124] E-Nose Pty Ltd, Eveleigh NSW, Australia; Available: http://www.e-nose.info/about.html, October 21, 2019.

[125] Alpha MOS, Toulouse, France; Available: https:// www.alpha-mos.com/, October 21, 2019.

[126] RoboScientific, Cambridgeshire, UK; Availalbe: http://www.roboscientific.com/, October 21, 2019.

[127] Aryballe, Grenoble, France; Available: https://aryballe. com/, October 21, 2019.

[128] Odotech, Montreal (Quebec) Canada; Available: http://www.odotech.com/en/, October 21, 2019.

[129] FIGARO, Osaka, Japan; Available: https://www.figaro. co.jp/en/, October 21, 2019.

[130] The eNose Company, Zutphen, The Netherlands; Available: https://www.enose.nl/, October 21, 2019.

[131] Y. Yano, K. Yokoyama, E. Tamiya, I. Karube. Direct evaluation of meat spoilage and the progress of aging using biosensors. Analytica Chimica Acta, vol.320, no. 2-3, pp. 269-276, 1996. DOI: 10.1016/0003-2670(95)00543-9.

[132] M. Garcia, M. Aleixandre, J. Gutierrez, M. C. Horrillo. Electronic nose for ham discrimination. Sensors and Actuators B: Chemical, vol.114, no.1, pp.418-422, 2006. DOI: $10.1016 /$ j.snb.2005.04.045.

[133] J. S. Vestergaard, M. Martens, P. Turkki. Application of an electronic nose system for prediction of sensory quality changes of a meat product (pizza topping) during storage. LWT-Food Science and Technology, vol.40, no.6, pp. 1095-1101, 2007. DOI: 10.1016/j.lwt.2006.06.008.

[134] N. El Barbri, E. Llobet, N. El Bari, X. Correig, B. Bouchikhi. Electronic nose based on metal oxide semiconductor sensors as an alternative technique for the spoilage classification of red meat. Sensors, vol. 8, no.1, pp. $142-156,2008$. DOI: $10.3390 / \mathrm{s} 8010142$.

[135] X. Y. Tian, Q. Cai, Y. M. Zhang. Rapid classification of hairtail fish and pork freshness using an electronic nose based on the PCA method. Sensors, vol.12, no.1, pp. 260-277, 2012. DOI: 10.3390/s120100260.

[136] W. Wojnowski, T. Majchrzak, T. Dymerski, J. Gebicki, J. Namiesnik. Portable electronic nose based on electrochemical sensors for food quality assessment. Sensors, vol.17, no.12, Article number 2715, 2017. DOI: $10.3390 / \mathrm{s} 17122715$.

[137] F. Li, J. Sun, X. Y. Huang, Y. Li, C. D. Zhang, X. R. Su. Establish poultry meat detection and identification model with the electronic nose. Journal of Chinese Institute of Food Science and Technology, vol.14, no. 2, pp. 255-260, 2014. (in Chinese)

[138] Z. Haddi, N. El Barbri, K. Tahri, M. Bougrini, N. El Bari, E. Llobetd, B. Bouchikhi. Instrumental assessment of red meat origins and their storage time using electronic sensing systems. Analytical Methods, vol.7, no.12, pp. 5193-5203, 2015. DOI: 10.1039/C5AY00572H.

[139] K. Timsorn, T. Thoopboochagorn, N. Lertwattanasakul, C. Wongchoosuk. Evaluation of bacterial population on chicken meats using a briefcase electronic nose. Biosys- 
tems Engineering, vol.151, pp.116-125, 2016. DOI: 10.1016/j.biosystemseng.2016.09.005.

[140] N. Christodoulides, M. P. McRae, G. W. Simmons, S. S. Modak, J. T. McDevitt. Sensors that learn: The evolution from taste fingerprints to patterns of early disease detection. Micromachines, vol.10, no. 4, Article number 251, 2019. DOI: $10.3390 / \mathrm{mi} 10040251$.

[141] D. R. Wijaya, R. Sarno, E. Zulaika, S. I. Sabila. Development of mobile electronic nose for beef quality monitoring. Procedia Computer Science, vol.124, pp.728-735, 2017. DOI: $10.1016 /$ j.procs.2017.12.211.

[142] E. Gorska-Horczyczak, I. Wojtasik-Kalinowska, D. Guzek, D. W. Sun, A. Wierzbicka. Differentiation of chillstored and frozen pork necks using electronic nose with ultra-fast gas chromatography. Journal of Food Process Engineering, vol.40, no. 5, Article number e12540, 2017. DOI: $10.1111 /$ jfpe. 12540 .

[143] M. Ghasemi-Varnamkhasti, S. S. Mohtasebi, M. Siadat, S. Balasubramanian. Meat quality assessment by electronic nose (machine olfaction technology). Sensors, vol. 9, no. 8, pp.6058-6083, 2009. DOI: 10.3390/ s90806058.

[144] W. S. Jia, G. Liang, Y. L. Wang, J. H. Wang. Electronic noses as a powerful tool for assessing meat quality: A mini review. Food Analytical Methods, vol.11, no.10, pp. 2916-2924, 2018. DOI: 10.1007/s12161-018-1283-1.

[145] M. Rivai, F. Budiman, D. Purwanto, J. Simamora. Meat freshness identification system using gas sensor array and color sensor in conjunction with neural network pattern recognition. Journal of Theoretical and Applied Information Technology, vol. 96, no. 12, pp.3861-3872, 2018.

[146] J. Z. Tan, W. L. Kerr. Determining degree of roasting in cocoa beans by artificial neural network (ANN)-based electronic nose system and gas chromatography/mass spectrometry (GC/MS). Journal of the Science of Food and Agriculture, vol. 98, no. 10, pp. 3851-3859, 2018. DOI: $10.1002 /$ jsfa. 8901 .

[147] D. Compagnone, M. Faieta, D. Pizzoni, C. Di Natale, R. Paolesse, T. Van Caelenberg, B. Beheydt, P. Pittia. Quartz crystal microbalance gas sensor arrays for the quality control of chocolate. Sensors and Actuators B: Chemical, vol. 207, pp.1114-1120, 2015. DOI: $10.1016 /$ j. snb.2014.10.049.

[148] L. F. Valdez, J. M. Gutierrez. Chocolate classification by an electronic nose with pressure controlled generated stimulation. Sensors, vol.16, no. 10, Article number 1745, 2016. DOI: $10.3390 / \mathrm{s} 16101745$.

[149] Z. B. Shi, T. Yu, Q. Zhao, Y. Li, Y. B. Lan. Comparison of algorithms for an electronic nose in identifying liquors. Journal of Bionic Engineering, vol.5, no. 3, pp. 253-257, 2008. DOI: 10.1016/S1672-6529(08)60032-3.

[150] S. T. Roweis, L. K. Saul. Nonlinear dimensionality reduction by locally linear embedding. Science, vol. 290, no. 5500, pp. 2323-2326, 2000. DOI: 10.1126/science.290. 5500.2323 .

[151] M. M. Macias, J. E. Agudo, A. G. Manso, C. J. G. Orellana, H. M. G. Velasco, R. G. Caballero. A compact and low cost electronic nose for aroma detection. Sensors, vol. 13, no. 5, pp.5528-5541, 2013. DOI: 10.3390/ s130505528.

[152] C. Sanchez, J. Lozano, J. P. Santos, A. Azabal, S. RuizValdepenas. Discrimination of aromas in beer with electronic nose. In Proceedings of Spanish Conference on
Electron Devices, IEEE, Salamanca, Spain, 2018. DOI: 10.1109/CDE.2018.8596955.

[153] C. Pornpanomchai, N. Suthamsmai. Beer classification by electronic nose. In Proceedings of International Conference on Wavelet Analysis and Pattern Recognition, IEEE, Hong Kong, China, pp.333-338, 2008. DOI: 10.1109/ICWAPR.2008.4635799.

[154] M. Siadat, E. Losson, M. Ghasemi-Varnamkhasti, S. S. Mohtasebi. Application of electronic nose to beer recognition using supervised artificial neural networks. In Proceedings of International Conference on Control, Decision and Information Technologies, IEEE, Metz, France, pp.640-645, 2014. DOI: 10.1109/CoDIT.2014. 6996971.

[155] A. Quarto, D. Soldo, F. Di Lecce, A. Giove, V. Di Lecce, A. Castronovo. Electronic nose for evaluating water use in beer production. In Proceedings of ISOCS/IEEE International Symposium on Olfaction and Electronic Nose, IEEE, Montreal, Canada, 2017. DOI: 10.1109/ISOEN. 2017.7968857.

[156] R. Dutta, E. L. Hines, J. W. Gardner, K. R. Kashwan, M. Bhuyan. Tea quality prediction using a tin oxide-based electronic nose: An artificial intelligence approach. Sensors and Actuators B: Chemical, vol.94, no.2, pp. 228-237, 2003. DOI: 10.1016/S0925-4005(03)00367-8.

[157] J. S. R. Jang, C. T. Sun, E. Mizutani. Neuro-fuzzy and Soft Computing: A Computational Approach to Learning and Machine Intelligence, Upper Saddle River, USA: Prentice-Hall, 1997.

[158] H. C. Yu, J. Wang, H. Xiao, M. Liu. Quality grade identification of green tea using the eigenvalues of PCA based on the E-nose signals. Sensors and Actuators B: Chemical, vol.140, no.2, pp.378-382, 2009. DOI: $10.1016 / \mathrm{j}$. snb.2009.05.008.

[159] L. Dutta, C. Talukdar, A. Hazarika, M. Bhuyan. A novel low-cost hand-held tea flavor estimation system. IEEE Transactions on Industrial Electronics, vol.65, no.6, pp. 4983-4990, 2018. DOI: 10.1109/TIE.2017.2772184.

[160] E. Bona, R. S. Optimized neural network for instant coffee classification through an electronic nose. International Journal of Food Engineering, vol.7, no.6, pp. 1556-3758, 2011. DOI: 10.2202/1556-3758.2002.

[161] G. Y. F. Makimori, E. Bona. Commercial instant coffee classification using an electronic nose in tandem with the ComDim-LDA approach. Food Analytical Methods, vol.12, no.5, pp.1067-1076, 2019. DOI: 10.1007/s12161019-01443-5.

[162] K. J. Flambeau, W. J. Lee, J. Yoon. Discrimination and geographical origin prediction of washed specialty bourbon coffee from different coffee growing areas in Rwanda by using electronic nose and electronic tongue. Food Science and Biotechnology, vol.26, no.5, pp.1245-1254, 2017. DOI: $10.1007 / \mathrm{s} 10068-017-0168-1$.

[163] T. Majchrzak, W. Wojnowski, T. Dymerski, J. Gebicki, J. Namiesnik. Electronic noses in classification and quality control of edible oils: A review. Food Chemistry, vol.246, pp. 192-201, 2018. DOI: 10.1016/j.foodchem.2017.11.013.

[164] E. Choe, D. B. Min. Chemistry of deep-fat frying oils. Journal of Food Science, vol. 72, no. 5, pp. R77-R86, 2007. DOI: $10.1111 / \mathrm{j} .1750-3841.2007 .00352 . x$.

[165] F. Anwar, A. I. Hussain, S. Iqbal, M. I. Bhanger. Enhancement of the oxidative stability of some vegetable oils by blending with Moringa oleifera oil. Food Chem- 
istry, vol.103, no.4, pp.1181-1191, 2007. DOI: 10.1016/ j.foodchem.2006.10.023.

[166] T. Majchrzak, M. Lubinska, A. Rozanska, T. Dymerski, J. Gebicki, J. Namiesnik. Thermal degradation assessment of canola and olive oil using ultra-fast gas chromatography coupled with chemometrics. Monatshefte für Chemie-Chemical Monthly, vol.148, no.9, pp.1625-1630, 2017. DOI: $10.1007 /$ s00706-017-1968-y.

[167] E. Ordukaya, B. Karlik. Quality control of olive oils using machine learning and electronic nose. Journal of Food Quality, vol.2017, Article number 9272404, 2017. DOI: $10.1155 / 2017 / 9272404$.

[168] M. J. Oates, P. Fox, L. Sanchez-Rodriguez, A. A. Carbonell-Barrachina, A. Ruiz-Canales. DFT based classification of olive oil type using a sinusoidally heated, low cost electronic nose. Computers and Electronics in Agriculture, vol. 155, pp.348-358, 2018. DOI: 10.1016/j.compag. 2018.10.026.

[169] Y. Yin, Y. F. Hao, Y. Bai, H. C. Yu. A Gaussian-based kernel Fisher discriminant analysis for electronic nose data and applications in spirit and vinegar classification. Journal of Food Measurement and Characterization, vol.11, no.1, pp.24-32, 2017. DOI: 10.1007/s11694-0169367-3.

[170] R. S. Lima, G. C. Danielski, A. C. S. Pires. Mastitis detection and prediction of milk composition using gas sensor and electrical conductivity. Food and Bioprocess Technology, vol.11, no.3, pp.551-560, 2018. DOI: 10.1007/s11947-017-2029-6.

[171] M. J. Anand, V. Sridhar. Characterisation of gas sensor array for milk spoilage and diseases detection. International Journal of Electronics, Electrical and Computational System, vol. 7, no. 2, pp. 48-52, 2018.

[172] O. Canhoto, N. Magan. Electronic nose technology for the detection of microbial and chemical contamination of potable water. Sensors and Actuators B: Chemical, vol.106, no.1, pp.3-6, 2005. DOI: 10.1016/j.snb.2004. 05.029 .

[173] R. Palasuek, T. Seesa-ard, C. Kunarak, T. Kerdcharoen. Electronic nose for water monitoring: The relationship between wastewater quality indicators and odor. In Proceedings of the 12th International Conference on Electrical Engineering/Electronics, Computer, Telecommunications and Information Technology, IEEE, Hua Hin, Thailand, 2015. DOI: 10.1109/ECTICon.2015.7206961.

[174] A. Blanco-Rodriguez, V. F. Camara, F. Campo, L. Becheran, A. Duran, V. D. Vieira, H. de Melo, A. R. GarciaRamirez. Development of an electronic nose to characterize odours emitted from different stages in a wastewater treatment plant. Water Research, vol.134, pp.92-100, 2018. DOI: 10.1016/j.watres.2018.01.067.

[175] R. Chandler, A. Das, T. Gibson, R. Dutta. Detection of oil pollution in seawater: Biosecurity prevention using electronic nose technology. In Proceedings of the 31st IEEE International Conference on Data Engineering Workshops, IEEE, Seoul, South Korea, pp.98-100, 2015. DOI: 10.1109/ICDEW.2015.7129554.

[176] A. C. Bastos, N. Magan. Potential of an electronic nose for the early detection and differentiation between streptomyces in potable water. Sensors and Actuators B: Chemical, vol.116, no.1-2, pp.151-155, 2006. DOI: 10.1016/j.snb.2005.11.073.

[177] A. D. Wilson. Diverse applications of electronic-nose technologies in agriculture and forestry. Sensors, vol. 13, no. 2, pp. 2295-2348, 2013. DOI: 10.3390/s130202295.

[178] L. Y. Chen, D. M. Wong, C. Y. Fang, C. I. Chiu, T. I. Chou, C. C. Wu, S. W. Chiu, K. T. Tang. Development of an electronic-nose system for fruit maturity and quality monitoring. In Proceedings of IEEE International Conference on Applied System Invention, IEEE, Chiba, Japan, pp. 1129-1130, 2018. DOI: 10.1109/ICASI.2018.8394481.

[179] L. Y. Chen, C. C. Wu, T. I. Chou, S. W. Chiu, K. T. Tang. Development of a dual MOS Electronic nose/camera system for improving fruit ripeness classification. Sensors, vol. 18, no. 10, Article number 3256, 2018. DOI: 10. $3390 / \mathrm{s} 18103256$.

[180] G. James, D. Witten, T. Hastie, R. Tibshirani. An Introduction to Statistical Learning: with Applications in $R$, New York, USA: Springer, 2013.

[181] Y. M. Ren, H. S. Ramaswamy, Y. Li, C. L. Yuan, X. L. Ren. Classification of impact injury of apples using electronic nose coupled with multivariate statistical analyses. Journal of Food Process Engineering, vol.41, no. 5, Article number e12698, 2018. DOI: 10.1111/jfpe.12698.

[182] E. Gobbi, M. Falasconi, G. Zambotti, V. Sberveglieri, A. Pulvirenti, G. Sberveglieri. Rapid diagnosis of Enterobacteriaceae in vegetable soups by a metal oxide sensor based electronic nose. Sensors and Actuators B: Chemical, vol.207, pp.1104-1113, 2015. DOI: 10.1016/j.snb.2014. 10.051 .

[183] L. Dentoni, L. Capelli, S. Sironi, R. Del Rosso, S. Zanetti, M. Della Torre. Development of an electronic nose for environmental odour monitoring. Sensors, vol.12, no.11, pp. 14363-14381, 2012. DOI: 10.3390/s121114363.

[184] S. Q. Cui, E. A. A. Inocente, H. P. Zhu, N. Acosta, P. P. Ling, H. M. Keener. Development of portable e-nose system for early diagnosis of insect-stressed tomato plants. In Proceedings of Annual International Meeting, American Society of Agricultural and Biological Engineers, St. Joseph, USA, 2018. DOI: 10.13031/aim.201800990.

[185] S. S. Lin, X. M. Zhang. A rapid and novel method for predicting nicotine alkaloids in tobacco through electronic nose and partial least-squares regression analysis. Analytical Methods, vol.8, no.7, pp.1609-1617, 2016. DOI: 10.1039/C5AY02257F.

[186] D. H. Luo, H. G. Hosseini, J. R. Stewart. Application of ANN with extracted parameters from an electronic nose in cigarette brand identification. Sensors and Actuators B: Chemical, vol.99, no.2-3, pp.253-257, 2004. DOI: 10.1016/j.snb.2003.11.022.

[187] M. Bassetti, G. Poulakou, E. Ruppe, E. Bouza, S. J. Van Hal, A. Brink. Antimicrobial resistance in the next 30 years, humankind, bugs and drugs: A visionary approach. Intensive Care Medicine, vol.43, no.10, pp.1464-1475, 2017. DOI: $10.1007 / \mathrm{s} 00134-017-4878-x$.

[188] A. D. Wilson. Electronic-nose devices-potential for noninvasive early disease-detection applications. Annals of Clinical Case Reports, vol. 2, Article number 1401, 2017.

[189] G. Santini, N. Mores, A. Penas, R. Capuano, C. Mondino, A. Trove, F. Macagno, G. Zini, P. Cattani, E. Martinelli, A. Motta, G. Macis, G. Ciabattoni, P. Montuschi. Electronic nose and exhaled breath NMR-based metabolomics applications in airways disease. Current Topics in Medicinal Chemistry, vol. 16, no. 14, pp. 1610-1630, 2016. DOI: $10.2174 / 1568026616666151223113540$. 
[190] P. Montuschi, N. Mores, A. Trove, C. Mondino, P. J. Barnes. The electronic nose in respiratory medicine. Respiration, vol. 85 , no. 1, pp. $72-84$, 2013. DOI: $10.1159 /$ 000340044.

[191] A. Voss, V. Baier, R. Reisch, K. von Roda, P. Elsner, H. Ahlers, G. Stein. Smelling renal dysfunction via electronic nose. Annals of Biomedical Engineering, vol. 33, no.5, pp. 656-660, 2005. DOI: 10.1007/s10439-005-1438-2.

[192] J. Vorobioff, E. Videla, N. Boggio, O. D. Salomon, A. Lamagna, C. A. Rinaldi. Laser vaporization e-nose method for the detection of transmitter of chagas disease. Sensors and Actuators B: Chemical, vol.257, pp.200-206, 2018. DOI: $10.1016 /$ j.snb.2017.10.143.

[193] H. M. Saraoglu, B. Edin. E-nose system for anesthetic dose level detection using artificial neural network. Journal of Medical Systems, vol.31, no.6, pp.475-482, 2007. DOI: $10.1007 / \mathrm{s} 10916-007-9087-7$.

[194] J. J. More. The levenberg-marquardt algorithm: Implementation and theory. Numerical Analysis, G. A. Watson, Ed., Berlin, Germany: Springer, pp. 105-116, 1978. DOI: $10.1007 /$ BFb0067700.

[195] J. A. Covington, E. W. Westenbrink, N. Ouaret, R. Harbord, C. Bailey, N. OS'Connell, J. Cullis, N. Williams, C. U. Nwokolo, K. D. Bardhan, R. P. Arasaradnam. Application of a novel tool for diagnosing bile acid diarrhoea. Sensors, vol.13, no.9, pp.11899-11912, 2013. DOI: $10.3390 / \mathrm{s} 130911899$.

[196] A. K. Pavlou, N. Magan, C. McNulty, J. Jones, D. Sharp, J. Brown, A. P. Turner. Use of an electronic nose system for diagnoses of urinary tract infections. Biosensors and Bioelectronics, vol.17, no.10, pp.893-899, 2002. DOI: 10.1016/S0956-5663(02)00078-7.

[197] V. S. Kodogiannis, J. N. Lygouras, A. Tarczynski, H. S. Chowdrey. Artificial odor discrimination system using electronic nose and neural networks for the identification of urinary tract infection. IEEE Transactions on Information Technology in Biomedicine, vol.12, no.6, pp. 707-713, 2008. DOI: 10.1109/TITB.2008.917928.

[198] W. Wojnowski, T. Dymerski, J. Gebicki, J. Namiesnik. Electronic noses in medical diagnostics. Current Medicinal Chemistry, vol.26, no.1, pp.197-215, 2019. DOI: 10.2174/0929867324666171004164636.

[199] W. Miekisch, S. Kischkel, A Sawacki, T. Liebau, M. Mieth, J. K. Schubert. Impact of sampling procedures on the results of breath analysis. Journal of Breath Research, vol.2, no. 2, Article number 026007, 2008. DOI: 10.1088/1752-7155/2/2/026007.

[200] S. M. Abbott, J. B. Elder, P. Spanel, D. Smith. Quantification of acetonitrile in exhaled breath and urinary headspace using selected ion flow tube mass spectrometry. International Journal of Mass Spectrometry, vol. 228 , no. $2-3$, pp.655-665, 2003. DOI: 10.1016/S13873806(03)00212-4.

[201] T. Majchrzak, W. Wojnowski, G. Piotrowicz, J. Gebicki, J. Namiesnik. Sample preparation and recent trends in volatolomics for diagnosing gastrointestinal diseases. TrAC Trends in Analytical Chemistry, vol.108, pp. 38-49, 2018. DOI: 10.1016/j.trac.2018.08.020.

[202] A. Amann, G. Poupart, S. Telser, M. Ledochowski, A. Schmid, S. Mechtcheriakov. Applications of breath gas analysis in medicine. International Journal of Mass Spectrometry, vol.239, no.2-3, pp.227-233, 2004. DOI: 10.1016/j.ijms.2004.08.010.
[203] S. Dragonieri, R. Schot, B. J. Mertens, S. Le Cessie, S. A. Gauw, A. Spanevello, O. Resta, N. P. Willard, T. J. Vink, K. F. Rabe, E. H. Bel, P. J. Sterk. An electronic nose in the discrimination of patients with asthma and controls. Journal of Allergy and Clinical Immunology, vol.120, no. 4, pp. 856-862, 2007. DOI: 10.1016/j.jaci.2007.05.043.

[204] S. Dragonieri, V. N. Quaranta, P. Carratu, T. Ranieri, O. Resta. Exhaled breath profiling by electronic nose enabled discrimination of allergic rhinitis and extrinsic asthma. Biomarkers, vol.24, no.1, pp.70-75, 2019. DOI: 10.1080/1354750X.2018.1508307.

[205] N. Fens, A. H. Zwinderman, M. P. van der Schee, S. B. de Nijs, E. Dijkers, A. C. Roldaan, D. Cheung, E. H. Bel, P. J. Sterk. Exhaled breath profiling enables discrimination of chronic obstructive pulmonary disease and asthma. American Journal of Respiratory and Critical Care Medicine, vol. 180, no.11, pp.1076-1082, 2009. DOI: 10. 1164/rccm.200906-0939OC.

[206] D. M. Wong, C. Y. Fang, L. Y. Chen, C. I. Chiu, T. I. Chou, C. C. Wu, S. W. Chiu, K. T. Tang. Development of a breath detection method based E-nose system for lung cancer identification. In Proceedings of IEEE International Conference on Applied System Invention, IEEE, Chiba, Japan, pp. 1119-1120, 2018. DOI: 10.1109/ICASI. 2018.8394477.

[207] R. van de Goor, M. van Hooren, A. M. Dingemans, B. Kremer, K. Kross. Training and validating a portable electronic nose for lung cancer screening. Journal of Thoracic Oncology, vol.13, no.5, pp.676-681, 2018. DOI: 10.1016/j.jtho.2018.01.024.

[208] N. Leunis, M. L. Boumans, B. Kremer, S. Din, E. Stobberingh, A. G. H. Kessels, K. W. Kross. Application of an electronic nose in the diagnosis of head and neck cancer. Laryngoscope, vol.124, no. 6, pp. 1377-1381, 2014. DOI: 10.1002/lary.24463.

[209] A. K. Pavlou, N. Magan, J. M. Jones, J. Brown, P. Klatser, A. P. F. Turner. Detection of Mycobacterium tuberculosis (TB) in vitro and in situ using an electronic nose in combination with a neural network system. Biosensors and Bioelectronics, vol.20, no.3, pp.538-544, 2004. DOI: 10.1016/j.bios.2004.03.002.

[210] R. Fend, A. H. J. Kolk, C. Bessant, P. Buijtels, P. R. Klatser, A. C. Woodman. Prospects for clinical application of electronic-nose technology to early detection of mycobacterium tuberculosis in culture and sputum. Journal of Clinical Microbiology, vol.44, no.6, pp. 2039-2045, 2006. DOI: 10.1128/JCM.01591-05.

[211] M. Bruins, Z. Rahim, A. Bos, W. W. J. van de Sande, H. P. Endtz, A. van Belkum. Diagnosis of active tuberculosis by e-nose analysis of exhaled air. Tuberculosis, vol.93, no. 2, pp.232-238, 2013. DOI: 10.1016/j.tube.2012. 10.002 .

[212] E. I. Mohamed, E. Bruno, R. Linder, M. Alessandrini, A. Di Girolamo, S. J. Pöppl, A. Puija, A. De Lorenzo. A novel method for diagnosing chronic rhinosinusitis based on an electronic nose. Anales Otorrinolaringologicos IberoAmericanos, vol.30, no. 5, pp.447-457, 2003.

[213] E. R. Thaler, D. D. Lee, C. W. Hanson. Diagnosis of rhinosinusitis with a colorimetric sensor array. Journal of Breath Research, vol.2, no.3, Article number 037016, 2008. DOI: $10.1088 / 1752-7155 / 2 / 3 / 037016$.

[214] B. Enderby, D. Smith, W. Carroll, W. Lenney. Hydrogen cyanide as a biomarker for Pseudomonas aeruginosa in the breath of children with cystic fibrosis. Pediatric Pul- 
monology, vol. 44, no. 2, pp. 142-147, 2009. DOI: 10.1002/ ppul.20963.

[215] C. M. H. H. T. Robroeks, J. J. B. N. van Berkel, J. W. Dallinga, Q. Jobsis, L. J. I. Zimmermann, H. J. E. Hendriks, M. F. M. Wouters, C. P. M. van der Grinten, K. D. G. van de Kant, F. J. van Schooten, E. Dompeling. Metabolomics of volatile organic compounds in cystic fibrosis patients and controls. Pediatric Research, vol.68, no. 1 , pp. $75-80, \quad 2010$. DOI: 10.1203/00006450201011001-00143.

[216] Z. J. Cheng, G. Warwick, D. H. Yates, P. S. Thomas. An electronic nose in the discrimination of breath from smokers and non-smokers: A model for toxin exposure. Journal of Breath Research, vol.3, no.3, Article number 036003, 2009. DOI: 10.1088/1752-7155/3/3/036003.

[217] T. Saidi, T. G. Welearegay, O. Zaim, O. G. Leon, R. Ionescu, N. E. Bari, B. Bouchikhi. Ability of discrimination of breath from smoker and non-smoker volunteers by using an electronic nose based on WO3 nanowires and $\mathrm{SnO} 2$ sensors. In Proceedings of International Symposium on Olfaction and Electronic Nose, ISOEN, Montreal, Canada, pp. 1-3, 2017.

[218] A. Voss, K. Witt, T. Kaschowitz, W. Poitz, A. Ebert, P. Roser, K. J. Bär. Detecting cannabis use on the human skin surface via an electronic nose system. Sensors, vol.14, no.7, pp.13256-13272, 2014. DOI: 10.3390/ s140713256.

[219] T. Straume, D. J. Loftus, J. Li, M. A. Coleman, C. E. Davis, K. A. McMonigal, M. Piccini, A. K. Singh. Biomarker-detection technologies for comprehensive medical diagnosis during deep-space missions. Recent Patents on Space Technology, vol.3, no.1, pp.13-23, 2013. DOI: $10.2174 / 18776116112029990009$.

[220] T. Wasilewski, D. Migon, J. Gebicki, W. Kamysz. Critical review of electronic nose and tongue instruments prospects in pharmaceutical analysis. Analytica Chimica Acta, vol. 1077, pp. 14-29, 2019. DOI: 10.1016/j.aca.2019. 05.024 .

[221] Z. Haddi, A. Amari, H. Alami, N. El Bari, E. Llobet, B. Bouchikhi. A portable electronic nose system for the identification of cannabis-based drugs. Sensors and Actuators B: Chemical, vol.155, no.2, pp.456-463, 2011. DOI: 10.1016/j.snb.2010.12.047.

[222] S. H. Zheng, W. G. Ren, L. F. Huang. Geoherbalism evaluation of radix Angelica sinensis based on electronic nose. Journal of Pharmaceutical and Biomedical Analysis, vol.105, pp.101-106, 2015. DOI: 10.1016/j.jpba.2014. 10.033 .

[223] R. Munoz, E. C. Sivret, G. Parcsi, R. Lebrero, X. G. Wang, I. H. Suffet, R. M. Stuetz. Monitoring techniques for odour abatement assessment. Water Research, vol.44, no. 18, pp.5129-5149, 2010. DOI: 10.1016/j.watres.2010. 06.013 .

[224] H. Singh, V. B. Raj, J. Kumar, U. Mittal, M. Mishra, A. T. Nimal, M. U. Sharma, V. Gupta. Metal oxide SAW enose employing PCA and ANN for the identification of binary mixture of DMMP and methanol. Sensors and Actuators B: Chemical, vol.200, pp.147-156, 2014. DOI: 10.1016/j.snb.2014.04.065.

[225] B. Mumyakmaz, K. Karabacak. An E-nose-based indoor air quality monitoring system: Prediction of combustible and toxic gas concentrations. Turkish Journal of Electrical Engineering \& Computer Sciences, vol.23, no.3, pp. 729-740, 2015. DOI: 10.3906/elk-1304-210.

[226] J. He, L. Y. Xu, P. Wang, Q. Wang. A high precise e-nose for daily indoor air quality monitoring in living environment. Integration, vol.58, pp.286-294, 2017. DOI: 10.1016/j.vlsi.2016.12.010.

[227] G. Jasinski, L. Wozniak, P. Kalinowski, P. Jasinski. Evaluation of the electronic nose used for monitoring environmental pollution. In Proceedings of XV International Scientific Conference on Optoelectronic and Electronic Sensors, IEEE, Warsaw, Poland, 2018. DOI: 10.1109/ COE.2018.8435146.

[228] M. Ismail, A. A. Majeed, Y. A. Albastaki. A framework for an artificial-neural-network-based electronic nose. Electronic Nose Technologies and Advances in Machine Olfaction, Y. A. Albastaki, F. Albalooshi, Eds., IGI Global, Pennsylvania, USA, pp. 1-24, 2018. DOI: 10.4018/9781-5225-3862-2.ch001.

[229] M. Sreelatha, G. M. Nasira. Surface acoustic wave e-nose sensor based pattern generation and recognition of toxic gases using artificial neural network techniques. Nature Inspired Computing, B. Panigrahi, M. Hoda, V. Sharma, S. Goel, Eds., Singapore: Springer, pp. 135-145, 2018. DOI: $10.1007 / 978-981-10-6747-1 \_16$.

[230] Y. Bengio, Y. LeCun. Scaling learning algorithms towards AI. Large-scale Kernel Machines, L. Bottou, O. Chapelle, D. DeCoste, J. Weston, Eds., MIT Press, Cambridge, USA, pp. 1-41, 2007.

[231] J. Gebicki, T. Dymerski, J. Namiesnik. Monitoring of odour nuisance from landfill using electronic nose. Chemical Engineering Transactions, vol.40, pp.85-90, 2014. DOI: $10.3303 / \mathrm{CET} 1440015$.

[232] P. K. Sekhar, E. L. Brosha, R. Mukundan, F. H. Garzon. Chemical sensors for environmental monitoring and homeland security. Electrochemical Society Interface, vol. 19, no. 4, pp. 35-40, 2010. DOI: 10.1149/2.F04104if.

[233] J. Wang. Electrochemical sensing of explosives. In Counterterrorist Detection Techniques of Explosives, J. Yinon, Ed., Amsterdam, Netherlands: Elsevier, pp.91-107, 2007. DOI: 10.1016/B978-044452204-7/50023-7.

[234] M. A. A. Bakar, A. H. Abdullah, F. S. A. Saad, S. A. A. Shukor, A. A. A. Razak, M. H. Mustafa. Electronic nose calibration process for monitoring atmospheric hazards in confined space applications. Journal of Telecommunication, Electronic and Computer Engineering, vol.10, no. 1-14, pp. 15-19, 2018.

[235] S. Limbo, L. Torri, N. Sinelli, L. Franzetti, E. Casiraghi. Evaluation and predictive modeling of shelf life of minced beef stored in high-oxygen modified atmosphere packaging at different temperatures. Meat Science, vol. 84, no. 1, pp. 129-136, 2010. DOI: 10.1016/j.meatsci.2009. 08.035 .

[236] E. Poyatos-Racionero, J. V. Ros-Lis, J. L. Vivancos, R. Martinez-Manez. Recent advances on intelligent packaging as tools to reduce food waste. Journal of Cleaner Production, vol.172, pp.3398-3409, 2018. DOI: 10.1016/ j.jclepro.2017.11.075.

[237] Y. Wang, J. G. Xing, S. Qian. Selectivity enhancement in electronic nose based on an optimized DQN. Sensors, vol.17, no. 10, Article number 2356, 2017. DOI: 10. $3390 / \mathrm{s} 17102356$.

[238] F. Röck, N. Barsan, U. Weimar. Electronic nose: Current status and future trends. Chemical Reviews, vol.108, 
no. 2, pp. 705-725, 2008. DOI: 10.1021/cr068121q.

[239] A. U. Rehman, A. Bermak. Drift-insensitive features for learning artificial olfaction in e-nose system. IEEE Sensors Journal, vol.18, no. 17, pp. 7173-7182, 2018. DOI: 10.1109/JSEN.2018.2853674.

[240] A. Vergara, R. Calavia, R. M. Vazquez, A. Mozalev, A. Abdelghani, R. Huerta, E. H. Hines, E. Llobet. Multifrequency interrogation of nanostructured gas sensor arrays: A tool for analyzing response kinetics. Analytical Chemistry, vol.84, no.17, pp.7502-7510, 2012. DOI: 10.1021/ ac301506t.

[241] S. Ampuero, J. O. Bosset. The electronic nose applied to dairy products: A review. Sensors and Actuators B: Chemical, vol.94, no.1, pp.1-12, 2003. DOI: 10.1016/ S0925-4005(03)00321-6.

[242] C. Wongchoosuk, M. Lutz, T. Kerdcharoen. Detection and classification of human body odor using an electronic nose. Sensors, vol.9, no. 9, pp.7234-7249, 2009. DOI: $10.3390 / \mathrm{s} 90907234$.

[243] X. Z. Hong, J. Wang. Detection of adulteration in cherry tomato juices based on electronic nose and tongue: Comparison of different data fusion approaches. Journal of Food Engineering, vol.126, pp.89-97, 2014. DOI: 10.1016/j.jfoodeng.2013.11.008.

[244] M. S. Yao, W. X. Tang, G. E. Wang, B. Nath, G. Xu. MOF thin film-coated metal oxide nanowire array: Significantly improved chemiresistor sensor performance. Advanced Materials, vol.28, no.26, pp.5229-5234, 2016. DOI: $10.1002 /$ adma.201506457.

[245] R. Trivino, D. Gaibor, J. Mediavilla, A. V. Guarnan. Challenges to embed an electronic nose on a mobile robot. In Proceedings of IEEE ANDESCON, IEEE, Arequipa, Peru, pp. 1-4. DOI: 10.1109/ANDESCON.2016.7836251.

[246] S. Dragonieri, G. Pennazza, P. Carratu, O. Resta. Electronic nose technology in respiratory diseases. Lung, vol.195, no. 2, pp.157-165, 2017. DOI: $10.1007 / \mathrm{s} 00408-$ 017-9987-3.

[247] Z. Y. Ma, G. C. Luo, K. Qin, N. Wang, W. N. Niu. Online sensor drift compensation for e-nose systems using domain adaptation and extreme learning machine. Sensors, vol.18, no. 3, Article number 742, 2018. DOI: 10. $3390 / \mathrm{s} 18030742$.

[248] A. C. Romain, J. Nicolas. Long term stability of metal oxide-based gas sensors for e-nose environmental applications: An overview. Sensors and Actuators B: Chemical, vol. 146, no. 2, pp. 502-506, 2010. DOI: 10.1016/j.snb.2009. 12.027 .

[249] R. Laref, D. Ahmadou, E. Losson, M. Siadat. Orthogonal signal correction to improve stability regression model in gas sensor systems. Journal of Sensors, vol.2017, Article number 9851406, 2017. DOI: 10.1155/2017/9851406.

[250] I. Sayago, M. Aleixandre, J. P. Santos. Development of tin oxide-based nanosensors for electronic nose environmental applications. Biosensors, vol. 9, no. 1, Article number 21, 2019. DOI: 10.3390/bios9010021.

[251] R. Hoekstra, P. Blondeau, F. J. Andrade. Distributed electrochemical sensors: Recent advances and barriers to market adoption. Analytical and Bioanalytical Chemistry, vol.410, no. 17 , pp. $4077-4089,2018$. DOI: 10.1007/s00216-018-1104-9.

[252] C. Sanchez, J. P. Santos, J. Lozano. Use of electronic noses for diagnosis of digestive and respiratory diseases through the breath. Biosensors, vol.9, no.1, Article number 35, 2019. DOI: $10.3390 /$ bios9010035.

[253] P. Pelosi, J. Zhu, W. Knoll. From gas sensors to biomimetic artificial noses. Chemosensors, vol.6, no. 3, Article number 32, 2018. DOI: 10.3390/chemosensors6030032.

[254] M. Mirshahi, V. Partovi Nia, L. Adjengue. Automatic odor prediction for electronic nose. Journal of Applied Statistics, vol.45, no. 15 , pp. 2788-2799, 2018. DOI: 10.1080/02664763.2018.1441382.

[255] N. Lotfivand, V. Abdolzadeh, M. N. Hamidon. Artificial olfactory system with fault-tolerant sensor array. ISA Transactions, vol.63, pp.425-435, 2016. DOI: 10.1016/ j.isatra.2016.03.012.

[256] G. Magna, C. Di Natale, E. Martinelli. Self-repairing classification algorithms for chemical sensor array. Sensors and Actuators B: Chemical, vol.297, Article number 126721, 2019. DOI: 10.1016/j.snb.2019.126721.

[257] G. S. W. Hagler, R. Williams, V. Papapostolou, A. Polidori. Air quality sensors and data adjustment algorithms: When is it no longer a measurement? Environmental Science \& Technology, vol.52, no.10, pp. 5530-5531, 2018. DOI: 10.1021/acs.est.8b01826.

[258] P. Verma, S. Panda. Polymer selection approaches for designing electronic noses: A comparative study. Sensors and Actuators B: Chemical, vol.273, pp.365-376, 2018. DOI: $10.1016 /$ j.snb.2018.06.015.

[259] T. T. Sunil, S. Chaudhuri, V. Mishra. Optimal selection of SAW sensors for e-nose applications. Sensors and Actuators B: Chemical, vol.219, pp.238-244, 2015. DOI: 10.1016/j.snb.2015.04.107.

[260] E. Zubritsky. Product review: E-noses keep an eye on the future. Analytical Chemistry, vol.72, no.11, pp. 421A-426A, 2000. DOI: 10.1021/ac0028447.

[261] H. Y. Zhou, D. H. Luo, H. GholamHosseini, Z. Li, J. F. He. Identification of Chinese herbal medicines with electronic nose technology: Applications and challenges. Sensors, vol. 17, no. 5, Article number 1073, 2017. DOI: 10. $3390 /$ s17051073.

[262] J. Bergstra and Y. Bengio. Random search for hyperparameter optimization. Journal of Machine Learning Research, vol. 13, pp. 281-305, 2012.

[263] L. Zhang, D. Zhang, X. Yin, Y. Liu. A novel semi-supervised learning approach in artificial olfaction for E-Nose application. IEEE Sensors Journal, vol.16, no.12, pp. 4919-4931, 2016. DOI: 10.1109/JSEN.2016.2551743.

[264] M. Son, J. Y. Lee, H. J. Ko, T. H. Park. Bioelectronic nose: An emerging tool for odor standardization. Trends in Biotechnology, vol.35, no. 4, pp.301-307, 2017. DOI: 10.1016/j.tibtech.2016.12.007.

[265] T. Wasilewski, J. Gebicki, W. Kamysz. Bioelectronic nose: Current status and perspectives. Biosensors and Bioelectronics, vol.87, pp.480-494, 2017. DOI: 10.1016/ j.bios.2016.08.080.

[266] O. S. Kwon, H. S. Song, S. J. Park, S. H. Lee, J. H. An, J. W. Park, H. Yang, H. Yoon, J. Bae, T. H. Park, J. Jang. An ultrasensitive, selective, multiplexed superbioelectronic nose that mimics the human sense of smell. Nano Letters, vol.15, no.10, pp.6559-6567, 2015. DOI: 10. 1021/acs.nanolett.5b02286.

[267] T. Wasilewski, J. Gebicki, W. Kamysz. Advances in olfaction-inspired biomaterials applied to bioelectronic noses. Sensors and Actuators B: Chemical, vol.257, 
pp. 511-537, 2018. DOI: 10.1016/j.snb.2017.10.086.

[268] H. Breer. Olfactory receptors: Molecular basis for recognition and discrimination of odors. Analytical and Bioanalytical Chemistry, vol.377, no. 3, pp.427-433, 2003. DOI: 10.1007/s00216-003-2113-9.

[269] J. H. Lim, T. H. Park. Concept of bioelectronic nose. Bioelectronic Nose: Integration of Biotechnology and Nanotechnology, T. H. Park, Ed., Dordrecht, Netherlands: Springer, pp.1-22, 2014. DOI: 10.1007/978-94-017-86133_1.

$[270]$ S. H. Lee, T. H. Park. Recent advances in the development of bioelectronic nose. Biotechnology and Bioprocess Engineering, vol.15, no. 1, pp.22-29, 2010. DOI: 10. 1007/s12257-009-3077-1.

[271] F. Di Pietrantonio, M. Benetti, D.Cannata, E. Verona, A. Palla-Papavlu, J. M. Fernandez-Pradas, P. Serra, M. Staiano, A. Varriale, S. D'Auria. A surface acoustic wave bio-electronic nose for detection of volatile odorant molecules. Biosensors and Bioelectronics, vol.67, pp. 516-523, 2015. DOI: 10.1016/j.bios.2014.09.027.

[272] D. L. Hall, J. Llinas. An introduction to multisensor data fusion. Proceedings of the IEEE, vol.85, no.1, pp.6-23, 1997. DOI: $10.1109 / 5.554205$.

[273] C. X. Dai, X. Y. Huang, D. M. Huang, R. Q. Lv, J. Sun, Z. C. Zhang, M. Ma, J. H. Aheto. Detection of submerged fermentation of Tremella aurantialba using data fusion of electronic nose and tongue. Journal of Food Process Engineering, vol.42, no.3, Article number e13002, 2019. DOI: $10.1111 /$ jfpe. 13002 .

[274] S. Buratti, C. Malegori, S. Benedetti, P. Oliveri, G. Giovanelli. E-nose, e-tongue and e-eye for edible olive oil characterization and shelf life assessment: A powerful data fusion approach. Talanta, vol.182, pp.131-141, 2018. DOI: 10.1016/j.talanta.2018.01.096.

[275] N. T. Nguyen, S. T. Wereley, S. A. M. Shaegh. Fundamentals and applications of microfluidics, 3rd ed., Norwood, USA: Artech House, 2019.

[276] M. M. Montazeri, A. O'Brien, M. Hoorfar. Understanding microfluidic-based gas detectors: A numerical model to investigate fundamental sensor operation, influencing phenomena and optimum geometries. Sensors and Actuators B: Chemical, vol. 300, Article number 126904, 2019. DOI: $10.1016 /$ j.snb.2019.126904.

[277] M. Paknahad, A. Ahmadi, J. Rousseau, H. R. Nejad, M. Hoorfar. On-chip electronic nose for wine tasting: A digital microfluidic approach. IEEE Sensors Journal, vol.17, no. 14, pp.4322-4329, 2017. DOI: 10.1109/JSEN.2017. 2707525 .

[278] M. Otto. Chemometrics: Statistics and Computer Application in Analytical Chemistry, 3rd ed., Weinheim, Germany: John Wiley \& Sons, 2016. DOI: 10.1002/ 9783527699377

[279] Q. Peng, R. G. Tian, F. R. Chen, B. B. Li, H. G. Gao. Discrimination of producing area of Chinese Tongshan kaoliang spirit using electronic nose sensing characteristics combined with the chemometrics methods. Food Chemistry, vol.178, pp.301-305, 2015. DOI: $10.1016 / \mathrm{j}$. foodchem.2015.01.023.

[280] O. O. Ojuroye, R. N. Torah, A. O. Komolafe, S. P. Beeby. Embedded capacitive proximity and touch sensing flexible circuit system for electronic textile and wearable systems. IEEE Sensors Journal, vol.19, no.16, pp. 6975-6985, 2019. DOI: 10.1109/JSEN.2019.2911561.

[281] Q. Dong, L. P. Du, L. J. Zhuang, R. Li, Q. J. Liu, P. Wang. A novel bioelectronic nose based on brain-machine interface using implanted electrode recording in vivo in olfactory bulb. Biosensors and Bioelectronics, vol.49, pp.263-269, 2013. DOI: 10.1016/j.bios.2013. 05.035 .

[282] Z. H. Wen, W. B. Ye, X. J. Zhao, X. F. Pan. A novel 1D deep convolutional neural network based algorithm for mixture gases recognition. In Proceedings of the 61st International Midwest Symposium on Circuits and Systems, IEEE, Windsor, Canada, pp.893-896. DOI: 10.1109/MWSCAS.2018.8624038.

[283] X. J. Zhao, Z. H. Wen, X. F. Pan, W. B. Ye, A. Bermak. Mixture gases classification based on multi-label one-dimensional deep convolutional neural network. IEEE Access, vol.7, pp.12630-12637, 2019. DOI: 10.1109/ACCESS.2019.2892754.

[284] A. X. He, G. F. Wei, J. Yu, Z. N. Tang, Z. H. Lin, P. J. Wang. A novel dictionary learning method for gas identification with a gas sensor array. IEEE Transactions on Industrial Electronics, vol. 64, no. 12, pp.9709-9715, 2017. DOI: 10.1109/TIE.2017.2748034.

[285] W. Cao, C. M. Liu, P. F. Jia. Feature extraction and classification of citrus juice by using an enhanced L-KSVD on data obtained from electronic nose. Sensors, vol.19, no. 4, Article number 916, 2019. DOI: 10.3390/s19040916.

[286] Y. Oktar, M. Turkan. A review of sparsity-based clustering methods. Signal Processing, vol.148, pp. 20-30, 2018. DOI: 10.1016/j.sigpro.2018.02.010.

[287] L. Meier, S. Van De Geer, P. Buhlmann. The group lasso for logistic regression. Journal of the Royal Statistical Society Series B, vol.70, no.1, pp.53-71, 2008. DOI: 10.1111/j.1467-9868.2007.00627.x.

[288] J. Friedman, T. Hastie, R. Tibshirani. A note on the group lasso and a sparse group lasso, Available: https://arxiv.org/abs/1001.0736, December 17, 2019.

[289] J. Z. Huang, T. Zhang. The benefit of group sparsity. The benefit of group sparsity. The Annals of Statistics, vol. 38, no. 4, pp. 1978-2004, 2010. DOI: 10.1214/09-AOS778.

[290] J. Z. Huang, T. Zhang, D. Metaxas. Learning with structured sparsity. Journal of Machine Learning Research, vol. 12, pp. 3371-3412, 2011.

[291] Y. C. Eldar, M. Mishali. Robust recovery of signals from a structured union of subspaces. IEEE Transactions on Information Theory, vol.55, no.11, pp.5302-5316, 2009. DOI: $10.1109 /$ TIT.2009.2030471.

[292] E. Elhamifar, R. Vidal. Sparse subspace clustering. In Proceedings of Conference on Computer Vision and Pattern Recognition, IEEE, Miami, USA, pp.2790-2797, 2009.

[293] Y. Oktar, M. Turkan. K-polytopes: A superproblem of $k$ means. Signal, Image and Video Processing, vol.13, no. 6, pp. 1207-1214, 2019. DOI: 10.1007/s11760-019-01469-6.

[294] M. Elad. Sparse and Redundant Representations: From Theory to Applications in Signal and Image Processing, New York, USA: Springer, 2010.

[295] E. Okur, M. Turkan. A survey on automated melanoma detection. Engineering Applications of Artificial Intelligence, vol.73, pp. 50-67, 2018. DOI: 10.1016/j.engappai. 2018.04.028. 
[296] I. Rodriguez-Lujan, J. Fonollosa, R. Huerta. Machine learning methods in electronic nose analysis. In Proceedings of Advances and Applications of Data Science and Engineering, UPCommons, Madrid, Spain, 2016.

[297] J. Cepelewicz. Artificial intelligence has a strange new muse: Our sense of smell, [Online], Available: https:// www.wired.com/story/artificial-intelligence-has-astrange-new-muse-our-sense-of-smell/, August 04, 2019.

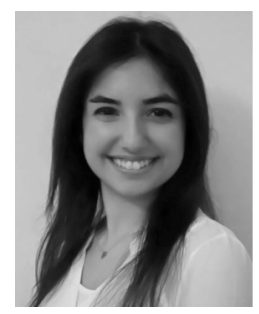

Diclehan Karakaya received the B.Sc. degree (with high honors) in electrical and electronics engineering from Izmir University of Economics, Turkey in 2017. She is currently a master student in electrical and electronics engineering at Izmir University of Economics, Turkey.

Her research interests include image processing, computer vision, machine learning and artificial intelligence.

E-mail: diclehankarakaya@gmail.com

ORCID iD: 0000-0002-7059-302X

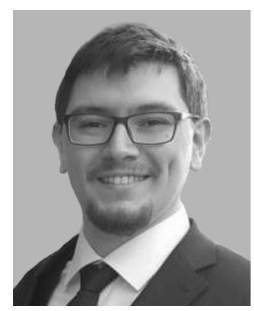

Oguzhan Ulucan received the B. Sc. degree (with honors) in electrical and electronics engineering from Izmir University of Economics, Turkey in 2017. He is currently a master student in electrical and electronics engineering at Izmir University of Economics, Turkey.

His research interests include image processing, computer vision, machine learning and artificial intelligence.

E-mail: oguzhan.ulucan.iz@gmail.com
ORCID iD: 0000-0003-2077-9691

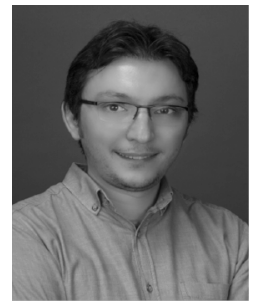

Mehmet Turkan received the B.Sc. (Hhons) degree in electrical and electronics engineering from Eskisehir Osmangazi University, Turkey in 2004, received the M.Sc. degree in electrical and electronics engineering from Bilkent University, Turkey in 2007. He received the Ph. D. degree in computer science from Bretagne Atlantique Research Center (INRIA) and University of Rennes 1, France in 2011. He is currently an assistant professor of electrical and electronics engineering at the Faculty of Engineering, Izmir University of Economics, Turkey. From January 2013 to July 2015, he was a full-time researcher at Technicolor Research \& Innovation Center, Cesson-Sevigne, France, where he was a post-doctoral researcher between November 2011 and December 2012. He was involved with the European Commission (EC) 6th Framework Program (FP6) Multimedia Understanding through Semantics, Computation and Learning Network of Excellence (MUSCLE-NoE), EC FP6 Integrated Three-Dimensional Television-Capture, Transmission, and Display Network of Excellence (3-DTV-NoE), and European UltraHD-4U research projects. He was the recipient of the HUAWEI Best Student Paper Award in the 2010 IEEE International Conference on Image Processing (IEEE-ICIP) and was a nominee for the Best Student Paper Award in the 2011 IEEE-ICIP.

His research interests include signal processing with an emphasis on image and video processing and compression, pattern recognition and classification, computer vision, machine learning and artificial intelligence.

E-mail: Mehmet.Turkan@gmail.com (Corresponding author) ORCID iD: 0000-0002-9780-9249 